\title{
The metallicity distribution of bulge clump giants in Baade's window ${ }^{\star} \star \star$
}

\author{
V. Hill ${ }^{1,2}$, A. Lecureur ${ }^{2}$, A. Gómez ${ }^{2}$, M. Zoccali ${ }^{4}$, M. Schultheis ${ }^{3}$, C. Babusiaux ${ }^{2}$, F. Royer ${ }^{2}$, B. Barbuy ${ }^{5}$, F. Arenou ${ }^{2}$, \\ D. Minniti ${ }^{4}$, and S. Ortolani ${ }^{7}$ \\ ${ }^{1}$ Université de Nice Sophia Antipolis, CNRS, Observatoire de la Côte d'Azur, bd. de l'Observatoire, BP 4229, 06304 Nice Cedex 4, \\ France \\ e-mail: Vanessa.Hill@oca.eu \\ 2 GEPI, Observatoire de Paris, CNRS UMR 8111, Université Paris Diderot, Place Jules Janssen, 92190 Meudon, France \\ 3 Observatoire de Besançon, CNRS UMR 6091, BP 1615, 25010 Besançon, France \\ 4 P. Universidad Católica de Chile, Departamento de Astronomía y Astrofísica, Casilla 306, Santiago 22, Chile \\ 5 Universidade de São Paulo, IAG, Rua do Matão 1226, São Paulo 05508-900, Brazil \\ 6 Universita di Padova,Vicolo dell’Osservatorio 5, 35122 Padova, Italy
}

Received 27 Novomber 2009 / Accepted 20 July 2011

\begin{abstract}
Aims. We seek to constrain the formation of the Galactic bulge by analysing the detailed chemical composition of a large sample of red clump stars in Baade's window. These stars were selected to minimise the contamination by other Galactic components, so they are good tracers of the bulge metallicity distribution in Baade's window, at least for stars more metal-rich than -1.5.

Methods. We used an automatic procedure to measure $[\mathrm{Fe} / \mathrm{H}]$ differentially with respect to the metal-rich star $\mu$ Leo in a sample of 219 bulge red clump stars from $R=20000$ resolution spectra obtained with FLAMES/GIRAFFE at the VLT. For a subsample of 162 stars, we also derived $[\mathrm{Mg} / \mathrm{H}]$ from spectral synthesis around the $\mathrm{Mg}$ I triplet at $\lambda 6319 \AA$.

Results. The Fe and $\mathrm{Mg}$ metallicity distributions are both asymmetric with median values of +0.16 and +0.21 , respectively. They show only a small proportion of stars at low metallicities, extending down to $[\mathrm{Fe} / \mathrm{H}]=-1.1$ or $[\mathrm{Mg} / \mathrm{H}]=-0.7$. The iron distribution is clearly bimodal, as revealed both by a deconvolution (from observational errors) and a Gaussian decomposition. The decomposition of the observed $\mathrm{Fe}$ and $\mathrm{Mg}$ metallicity distributions into Gaussian components yields two populations of equal sizes (50\% each): a metal-poor component centred on $[\mathrm{Fe} / \mathrm{H}]=-0.30$ and $[\mathrm{Mg} / \mathrm{H}]=-0.06$ with a large dispersion and a narrow metal-rich component centred on $[\mathrm{Fe} / \mathrm{H}]=+0.32$ and $[\mathrm{Mg} / \mathrm{H}]=+0.35$. The metal-poor component shows high $[\mathrm{Mg} / \mathrm{Fe}]$ ratios (around 0.3), while stars in the metal-rich component are found to have nearly solar ratios. Kinematical differences between the two components have also been found: the metal-poor component shows kinematics compatible with an old spheroid, while the metal-rich component is consistent with a population supporting a bar. In view of their chemical and kinematical properties, we suggest different formation scenarii for the two populations: a rapid formation time scale as an old spheroid for the metal-poor component (old bulge) and for the metal-rich component, a formation on a longer time scale driven by the evolution of the bar (pseudo-bulge). The observations are described well by a simple model consisting of two components: a simple closed box model to predict the metal-poor population contribution and a local thin disc metallicity distribution, shifted in metallicity, to represent the metal-rich population. The pseudo-bulge is compatible with its being formed from the inner thin disc, assuming high (but plausible) values of the gradients in the early Galactic disc.
\end{abstract}

Key words. Galaxy: bulge - Galaxy: formation - Galaxy: abundances - stars: abundances - stars: atmospheres

\section{Introduction}

The Milky Way bulge has been the subject of quite intense debates in the community, because its status has still not been fully established. With various stellar population characteristics similar to those of the central old spheroids found at the centre of earlier type galaxies, and others (mostly geometrical and kinematical) that point rather towards a very strong influence by a bar responsible for a pseudo-bulge, this bulge lies between these two types. The formation scenarios for bulges can be classified into three different types: (i) initial collapse of gas at early

^ Based on ESO-VLT observations during Paris Observatory FLAMES GTO 71.B-0196.

$\star \star$ Full Tables 1-3 are available at the CDS via anonymous ftp to cdsarc.u-strasbg.fr (130.79.128.5) or via

http://cdsarc.u-strasbg.fr/viz-bin/qcat?J/A+A/534/A80 times (see e.g., Eggen et al. 1962), (ii) merging subclumps, either through an early disc evolution (Noguchi 1999; Immeli et al. 2004), or through mergers (Aguerri et al. 2001; Scannapieco \& Tissera 2003; Nakasato \& Nomoto 2003), (iii) secular evolution of the Galactic disc (Combes \& Sanders 1981; Pfenniger \& Norman 1990; Raha et al. 1991). The merger scenario itself is similar to an early collapse scenario from the point of view of the formation characteristic time scale (early and fast). It has also been recently suggested that the Galactic bulge could be the result of both formation processes, with an old spheroid complemented by a bar-driven pseudo-bulge (Nakasato \& Nomoto 2003; Kormendy \& Kennicutt 2004; Gerhard 2006). The relative importance of the two processes (or even populations), however, remains to be determined.

The presence of a bar in the inner Galaxy was suggested by de Vaucouleurs (1964) from gas kinematics and has since 
Table 1. Sample identifications, BVRI OGLE, and JHK 2MASS photometry.

\begin{tabular}{lccccccccc}
\hline \hline ID & OGLE-ID & $\begin{array}{c}B \\
\mathrm{mag}\end{array}$ & $\begin{array}{c}V \\
\mathrm{mag}\end{array}$ & $\begin{array}{c}R \\
\mathrm{mag}\end{array}$ & $\begin{array}{c}I \\
\mathrm{mag}\end{array}$ & 2MASS-ID & $\begin{array}{c}J \\
\mathrm{mag}\end{array}$ & $\begin{array}{c}H \\
\mathrm{mag}\end{array}$ & $\begin{array}{c}K \\
\mathrm{mag}\end{array}$ \\
\hline BWc-1 & BUL-SC45 393125 & 20.985 & 18.587 & 16.838 & 15.010 & $\mathrm{~J} 18035033+3005324$ & 13.647 & 12.857 & 12.677 \\
BWc-2 & BUL-SC45 545749 & 20.434 & 18.830 & 17.191 & 15.392 & $\mathrm{~J} 18035671+3005378$ & 14.115 & 13.312 & 13.178 \\
BWc-3 & BUL-SC45 564840 & 20.179 & 18.689 & 16.906 & 15.144 & $\mathrm{~J} 18035461+3001064$ & 13.845 & 13.087 & 12.720 \\
BWc-4 & BUL-SC45 564857 & 19.593 & 18.240 & 16.760 & 15.161 & $\mathrm{~J} 18035531+3000576$ & 13.941 & 13.111 & 12.886 \\
BWc-5 & BUL-SC45 575542 & 21.116 & 18.750 & 16.982 & 15.175 & $\mathrm{~J} 18035592+2955439$ & 13.909 & 13.267 & 13.030 \\
BWc-6 & BUL-SC45 575585 & 19.497 & 18.238 & 16.744 & 15.069 & $\mathrm{~J} 18035640+2955122$ & 13.832 & 13.097 & 12.955 \\
BWc-7 & BUL-SC45 575585 & 19.497 & 18.238 & 16.744 & 15.069 & $\mathrm{~J} 18035640+2955122$ & 13.832 & 13.097 & 12.955 \\
BWc-8 & BUL-SC45 78255 & 20.702 & 18.616 & 16.972 & 15.163 & $\mathrm{~J} 18031236+3003596$ & 13.724 & 12.990 & 12.885 \\
BWc-9 & BUL-SC45 78271 & 20.588 & 18.527 & 16.903 & 15.131 & $\mathrm{~J} 18031656+3003517$ & 13.885 & 13.153 & 12.950 \\
BWc-10 & BUL-SC45 89589 & 19.790 & 18.213 & 16.695 & 15.058 & $\mathrm{~J} 18031877+3001101$ & 13.401 & 12.709 & 12.505 \\
$\ldots$ & $\ldots$ & $\ldots$ & $\ldots$ & $\ldots$ & $\ldots$ & & $\ldots$ & $\ldots$ & $\ldots$ \\
\hline
\end{tabular}

Notes. This table is available electronically at the CDS, including errors on the photometric bands.

been confirmed by numerous studies including infrared surface brightness map (e.g., Skrutskie et al. 2006), starcounts, redclump distances (e.g., Babusiaux \& Gilmore 2005; Nishiyama et al. 2006; Rattenbury et al. 2007), microlensing and stellar kinematics (e.g., Zhao et al. 1994; Howard et al. 2008, 2009). The boxy aspect of the bulge, detected in the infrared light profile (e.g., Dwek et al. 1995), also argues for a pseudo-bulge secularly evolved from the Galactic disc.

On the other hand, photometric studies in selected windows on the Galactic bulge in the visible and the near infrared soon made it apparent that the stellar populations in the bulge is old and metal-rich (Ortolani et al. 1995; Feltzing \& Gilmore 2000; Kuijken \& Rich 2002; Zoccali et al. 2003; Clarkson et al. 2008). Spectroscopically, Rich (1988) obtained one of the first metallicity distributions in Baade's window based on low-resolution spectra of M giants, followed by Ibata \& Gilmore (1995a,b) using K giants and Sadler et al. (1996) using red clump stars. All of them found a large metallicity dispersion. High-resolution spectra of a limited number of stars (10 to 20) in Baade's window (McWilliam \& Rich 1994; Fulbright et al. 2006, 2007), and more recently by our group (Zoccali et al. 2006; Lecureur et al. 2007) in a larger sample of 50 stars in four windows of the Galactic bulge, showed enhanced $[\alpha / \mathrm{Fe}]$, compatible with a fast chemical enrichment of the Galactic bulge. Both the chemical and age properties of stellar populations in the Galactic bulge thus point towards rapid bulge formation. Early combined metallicity and kinematics measurements have also pointed towards bulge formation through dissipational collapse (Minniti 1996).

More recently still, our group presented in Zoccali et al. (2008) the first metallicity distribution entirely based on highresolution spectra of a large sample of red giant branch stars in three fields of the Galactic bulge (close to the minor axis, at $b \sim-4,-6$, and $-12^{\circ}$ ). In this paper we show how determining metallicity distributions from a large $(>200)$ and almost uncontaminated sample of red clump stars in Baade's window, observed at high spectral resolution, can lead to significant improvements in our understanding of the origin and nature of the Galactic bulge. In particular, we show how using two independent elements (iron and magnesium) as metallicity tracers (and their $[\mathrm{Mg} / \mathrm{Fe}])$ reveals the nature of the stellar population. In Sect. 2, we present our target selection and observations with FLAMES on VLT, in Sect. 3 we detail the stellar parameter and elemental abundance measurement methods, while Sect. 4 examines the issues of sample representativity and contamination. Finally, in Sect. 5, we discuss the resulting $[\mathrm{Fe} / \mathrm{H}]$ and $[\mathrm{Mg} / \mathrm{H}]$ metallicity distributions and $[\mathrm{Mg} / \mathrm{Fe}]$ trends, show that

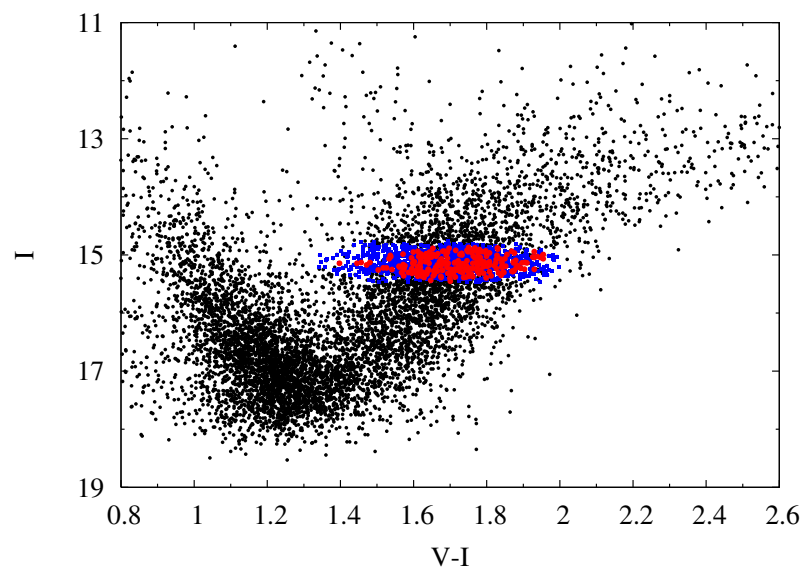

Fig. 1. $(I,(V-I))$ colour magnitude diagram for the Baade's window field BUL-SC45 from the OGLE II survey. The stars identified as red clump members by the OGLE survey are indicated in blue and the targets observed with FLAMES/GIRAFFE are indicated in red.

the sample can be separated into two distinct populations, and interpret these in the framework of various formation scenarios and chemical evolution models. Section 6 gives our conclusions from this work.

\section{Sample selection}

We selected a sample of stars in the Baade's window field BULSC45 from the OGLE II survey (Udalski et al. 1997; Paczynski et al. 1999) among the $\sim 1400$ stars identified as red clump members by the OGLE survey (see Fig. 1). The sample was first restricted to targets also present in the 2MASS (Skrutskie et al. 2006) and DENIS surveys (Epchtein et al. 1997), in order to expand the photometric coverage to the infrared, which is an asset for photometric temperature determination (see Sect. 3). From this subsample ( $\sim 800$ stars), a further restriction was applied in the $\left(K_{0},(J-K)_{0}\right)$ colour-magnitude diagram to lower a possible disc contamination: stars with $K_{0}<12$ and $(J-K)_{0}<0.5$ were rejected, as they lie in a locus where dwarfs are expected to extend. To do so, colours were dereddenned using the mean extinction value for the Baade's window $A_{V}=1.5$ (Paczynski et al. 1999) and the reddening law $A_{K} / A_{V}=0.089$ (Glass 1999). Stars with $16.5<V<17.2$ were then selected for observations with FLAMES-GIRAFFE, letting the fibre allocation procedure randomly pick the final selection of 228 stars (see Fig. 1). The sample OGLE and 2MASS identification and photometry are given 
in Table 1, available at the CDS in its entirety (the first lines of the table are reproduced in the printed version). To optimise the exposure time, the total sample was divided in two subsamples: (a) 114 stars with $16.5<V<16.9$ and (b) 114 stars with $16.9<V<17.2$. In each subsample, $\sim 16$ fibres were devoted to blank sky regions to allow for proper sky-subtraction. In total, 130 fibres were allocated in each of the two GIRAFFE fibre configurations (i.e., close to the maximum allowed).

\subsection{Observations}

The observations were performed during August 2003 with the ESO/VLT/FLAMES facility, as part of the guaranteed time observation programmes of the Paris Observatory (PI, A. Gómez). The spectra were obtained with the GIRAFFE spectrograph in Medusa mode using two high-resolution settings: HR13 et HR14, yielding resolving powers $R=22500$ and 28800 , respectively. Spectral coverage spans from 6120 to $6405 \AA$ for HR13 and from 6383 to $6626 \AA$ for HR14, and the total exposure times achieved were of $2 \mathrm{~h} 15$ and $3 \mathrm{~h} 00$ in HR13 for samples a) and b), respectively, and 4h30 and 6h00 in HR14 for samples a) and b), respectively. Using the FLAMES link to the UVES spectrograph, a subsample of 12 stars were simultaneously observed at higher resolutions $(R \sim 48000)$. This subsample has been previously described in Zoccali et al. (2006) and Lecureur et al. (2007).

The data reduction was carried out using the girBLDRS ${ }^{1}$ pipeline developed at the Geneva Observatory (Blecha et al. 2000) and includes cosmic-ray removal, bias subtraction, flatfield correction, individual spectral extraction, and accurate wavelength calibration based on simultaneous calibration exposures. Co-addition of the individual spectra and sky substraction was performed independently from the girBLDRS, using several IRAF tasks. For each setup, an "average sky" was made by combining the 16 sky fibre spectra, and was then subtracted from each target spectrum. All the spectra for each star were then coadded with a $\kappa$-sigma clipping to remove the cosmic rays.

\subsection{Line of sight sample contamination}

Since essentially all RGB stars experience the core heliumburning phase, red clump stars are excellent tracers of the metallicity distribution of a given stellar population. Moreover, as mentioned by Fulbright et al. (2006), the metallicity-dependent lifetime of the horizontal branch (HB) has a negligible effect on the metallicity distribution (MD). These authors estimated that an increase of 1 dex corresponds to an increase of $10 \%$ in the HB lifetime, leading to a correction of $-0.02 /-0.03$ on the MD. Red clump giants are also good candidates for sampling the bulge MD because the CMD region where they stand is known to be hardly contaminated by other Galactic components on the line of sight (Sadler et al. 1996; Fulbright et al. 2006). However, since stellar evolution models predict that the less metallic stars burn helium in their cores on the blue HB (and not in the clump), it is expected that there is a minimum metallicity that red clump stars can sample. We checked how our sample selection centred on the red clump would have missed metal-poor stars by the inspection of 9 and 12 Gyr old isochrones by Girardi et al. (2000) with various metallicities, in $J-K, V-I$, and $V-K$ bands, and compared them with our selection box (allowing for reddening). We thereby estimated that stars down to $[\mathrm{Fe} / \mathrm{H}] \geqslant-1.3$ or even -1.5 could be included in the sample selection box. However, because

\footnotetext{
${ }^{1}$ See http://girbldrs. sourceforge.net/
}
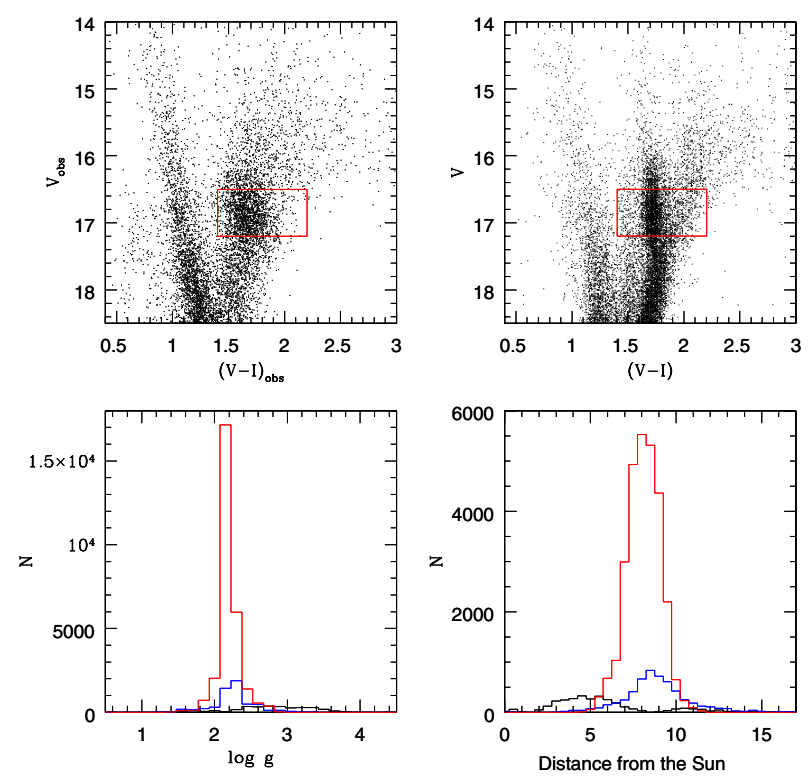

Fig. 2. Two upper panels: the $(V,(V-I))$ colour-magnitude diagrams of our field in Baade's window, as observed (left) and simulated by the Besançon model (right). The red boxes in these panels show the sample selection. The lower part of the figure presents the distributions in gravities (left) and distances (right) of model stars falling in the selection box, separated into their parent populations: bulge (red), thin disc (black), and thick disc (blue).

mass loss is poorly known and plays a major role in shaping the $\mathrm{HB}$, it is difficult to quantify the bias that is introduced by selecting the red clump for each metallicity bin. However, that the MD decreases smoothly up to our metal-poor limit suggests that we might be only losing a few stars. Furthermore, the comparison of our red clump-based MD to the one obtained by Zoccali et al. (2008) from a sample selected higher up on the RGB with no such built-in bias (see Sect. 4.1.4), shows no significant difference between the two samples on the metal-poor side (that however only reaches $[\mathrm{Fe} / \mathrm{H}] \sim-1.2$ to $-1.5 \mathrm{dex}$ ).

We estimated the expected contamination of our bulge red clump sample by foreground (and background) Galactic disc(s) stars and halo stars, using the latest population-synthesis Besançon model (Robin et al. 2003; Picaud \& Robin 2004). A $25^{\prime} \times 25^{\prime}$ field, centred on Baade's window, was simulated without including the reddening. The latter was added independently of the Besançon model with the following prescription based on best reproducing the locus of the CMD in this region (position of the foreground disc main sequence and red clump sequences): for stars located beyond $2 \mathrm{kpc}$ from the Sun (including the bulge stars) we applied the same reddening as used to compute the photometric temperature (Sect. 3, i.e., $E(B-V)=0.55$ ), while for stars within $2 \mathrm{kpc}$ from the Sun (mostly thin disc), a reddening proportional to the distance (to the Sun) was applied $\left(A_{V}=0.85 / \mathrm{kpc}\right)$. Further shifts of the order of $0.1-0.2 \mathrm{mag}$ had to be applied to both $V$ and $V-I$ in order to reproduce the position of the bulge clump in the observed CMD. As shown in Fig. 2, the simulated CMD shows a global morphology in agreement with the observed one, although the colour distribution of the simulated CMD is narrower than the observed, owing to the combined effects of our ignoring the differential reddening within the field and neglecting the photometric errors in the simulation.

In the sample selection box $(16.5<V<17.2$ et $1.4<V-I<$ 2.2 ), the total number of simulated stars is $\sim 31200$ stars, among which the vast majority are bulge stars (28383). The resulting 
contamination predicted by the Besançon model for our sample is $9.3 \%: 5.9 \%$ from the thick disc, $3.3 \%$ from the thin disc, and $0.1 \%$ from the halo. For the sample of 219 bulge clump stars, this corresponds to $7 \pm 3$ stars of the thin disc and $13 \pm 4$ stars of the thick disc. It can be appreciated from these low numbers (and from Fig. 2) that our red clump sample should be very little contaminated by any other intervening Galactic populations.

As illustrated in Fig. 2, while the thin disc contaminants lie in the foreground, thick disc contaminants are located in the very inner regions of the Galaxy, at distances similar to the bulge red clump population. The thick disc stars that enter our selection box are mainly giants, with temperatures and gravities similar to those of the red clump stars, and the vast majority are located beyond $7 \mathrm{kpc}$ from the Sun. For these thick-disc contaminating stars, the analysis method developed for the red clump sample and in particular the photometric gravity computed assuming that all the stars are located at a distance of $8 \mathrm{kpc}$, can also be applied and will provide an unbiased $[\mathrm{Fe} / \mathrm{H}]$ estimation for these stars. The thin disc stars, on the other hand, are also giants, but located in majority 3-6 kpc from the Sun, and therefore have significantly higher gravities than those of the red clump stars. This difference in $\log g$ reaches 0.4 to $1 \mathrm{dex}$, which in turn leads to biases in the derived $[\mathrm{Fe} / \mathrm{H}]$ (with our method assuming that all stars are located in the bulge, see Sect. 3) of approximately -0.2 to -0.25 dex. Whereas thick disc contaminants from our sample can be readily identifiable from their metallicity alone (assuming we know the metallicity distribution of the thick disc in the inner regions of the galaxy), thin disc contaminants are not expected to be easily identified from their position in the metallicity distribution.

The Besançon model makes a number of hypothesis, however, that may affect the estimates of contamination that we make here. One of them is that the thin disc density is assumed to decrease sharply in its central $2 \mathrm{kpc}$ (it has a hole in the centre), which has been adjusted (together with its scalelength) to reproduce star counts towards the bulge from the DENIS experiment, as explained in Picaud \& Robin (2004). This is the main reason for the low contamination by the thin disc predicted here: if the thin disc continued with an exponential profile all the way to the Galactic centre, the RGB and RC stars in the central 1$2 \mathrm{kpc}$ of the galaxy would contribute to this contamination. On the other hand, observational evidence of this hole is quite compelling from star counts, from the observation of a void in the gas surrounded by a molecular gas ring, and from the evidence for a stellar bar (Babusiaux \& Gilmore 2005; Nishiyama et al. 2006; Rattenbury et al. 2007) that would have swept away much of the matter in these inner regions. More important perhaps, because constraints on the density profile of the inner parts of the thick disc are very poor, the Besançon model assumes a simple exponential profile, with no hole in the centre. As a result, the major part of the predicted contamination of our sample originates in the inner regions of the thick disc, a prediction that is therefore rather poorly constrained.

Metallicity distributions assumed in the model also affect the position of the stars in each population in the colour-magnitude diagram, and can shift stars in or out of our observed selection box. The thin disc is assumed to host a metallicity gradient of $-0.07 \mathrm{dex} \mathrm{kpc}^{-1}$ (as constrained from the young stars and HII regions), which brings the innermost parts of the disc (around $2 \mathrm{kpc}$ from the centre, or $6 \mathrm{kpc}$ from the Sun) to supersolar metallicities. If the inner thin disc had no hole, thin disc RGB and RC stars in the central 1-2 kpc of the galaxy would have colours and magnitudes (and metallicities) very close to our sample's. However, the main-sequence counterpart of this younger population has so far eluded deep colour-magnitude diagrams of low-reddening regions (Clarkson et al. 2008). The thick disc contribution is again the most uncertain part of the Besançon model prediction, as there are currently no observational constraints regarding its radial metallicity gradient (or absence of). If the thick disc in the model had a radial gradient, it might bring a few more stars in the selection box. However, even with a metallicity gradient, it is unlikely that thick disc stars will contaminate the high-metallicity end of the sample, but would instead be found around metallicities of -0.5 or below.

\section{Determination of stellar parameters}

\subsection{Photometric parameters}

Photometric temperatures were determined from the $\mathrm{V}-\mathrm{K}, \mathrm{V}-\mathrm{H}$ and $V-J$ colours using the calibrations of Ramírez \& Meléndez (2005) and their extinction law with $E(B-V)=0.55$ to correct for reddening. The photometric gravities were calculated from the following equation:

$\log \left(\frac{g}{g_{\odot}}\right)=\log \left(\frac{M}{M_{\odot}}\right)-0.4\left(M_{\mathrm{Bol}, \odot}-M_{\mathrm{Bol}, *}\right)+4 \log \left(\frac{T_{\mathrm{eff}}}{T_{\mathrm{eff}, \odot}}\right)$

with $M_{\mathrm{Bol}, \odot}=4.72 \mathrm{mag}, T_{\mathrm{eff}, \odot}=5770 \mathrm{~K}, \log g_{\odot}=4.44 \mathrm{dex}$ for the Sun, and $M=0.8 M_{\odot}$ for the bulge stars. The bolometric magnitude was computed from the $V$ magnitude and the bolometric correction calibrations of Alonso et al. (1999) assuming that all stars were bulge members, at $8 \mathrm{kpc}$ from the Sun (Reid 1993).

Despite the use of infrared bands, both photometric $T_{\text {eff }}$ and $\log g$ values are affected by the differential reddening but show a different sensitivity. In Baade's window, the reddening spread is of the order of $0.15 \mathrm{mag}$ on $V-I$, leading to $\sim 200 \mathrm{~K}$ uncertainties on $T_{\text {eff }}$ and $\sim 0.05$ dex on $\log g$. Therefore the photometric temperature (mean value from the $V-K, V-H$, and $V-J$ colours) was only used as an initial value in the stellar parameter determination procedure (see Sect. 3.3). The main source of uncertainty in the $\log g$ determination is the distance of the star. Assuming that all sample stars are at the mean bulge distance to the Sun can lead to an error up to $0.25 \operatorname{dex}$ on $\log g$. However, on the whole sample, the previous uncertainty value remains lower than those expected from a $\log g$ deduced from the ionisation equilibrium. This is mainly due to uncertainties on the Fe II abundances coming from uncertainties in the equivalent widths measurements (continuum placement, blends) and on the $\log g f$ values of the Fe II lines (see Lecureur et al. 2007). At GIRAFFE resolution, the previous source of uncertainties becomes larger, and the photometric gravity was used as a final value. We also note that the $T_{\text {eff }}$ value influences the photometric $\log g$ : a change of $\sim 200 \mathrm{~K}$ can lead to changes on $\log g$ up to 0.15 dex for the cooler stars. Then, the $\log g$ value were recomputed at each step of the stellar parameter determination procedure taking the change in $T_{\text {eff }}$ into account.

\subsection{Spectroscopic parameters}

\subsubsection{EWs measurement, atmospheric models, and codes}

The equivalent widths (EWs) for selected $\mathrm{Fe}_{\mathrm{I}}$ and $\mathrm{Mg}$ lines were measured using the automatic code DAOSPEC ${ }^{2}$

2 DAOSPEC has been written by P. B. Stetson for the Dominion Astrophysical Observatory of the Herzberg Institute of Astrophysics, National Research Council, Canada. 
(Stetson \& Pancino 2008). The synthetic spectra were computed using the LTE spectral analysis code "turbospectrum" (described in Alvarez \& Plez 1998), and the abundances from EWs were derived using the Spite programs (Spite 1967, and subsequent improvements over the years) with for both codes the new MARCS models (Gustafsson et al. 2008).

\subsubsection{The iron line list}

The iron line list from Lecureur et al. (2007) was used for the stellar parameter determination. This line list was established in the following way. As a starting point, we used the line list from Zoccali et al. (2004) with log $g f$ values extracted from the NIST database $^{3}$ (Ralchenko et al. 2008). By computing synthetic spectra in the stellar parameters range of the red clump stars, we rejected the iron lines blended up to $10 \%$ of the equivalent width. Finally, for the remaining iron lines, the $\log g f$ values were adjusted so that each line gives an abundance of 0.30 dex from the EW measured on the observed spectrum of $\mu$ Leo with the following stellar parameters: $T_{\text {eff }}=4540 \mathrm{~K}, \log g=2.3$ dex, and $\xi=1.3 \mathrm{~km} \mathrm{~s}^{-1}$. The spectrum of $\mu$ Leo was obtained at the Canada-France-Hawaii Telescope with the ESPaDOnS spectropolarimeter (resolution $R \sim 80000$ and $S / N$ per pixel $\sim 500$ ) and processed using the "Libre ESpRIT" data reduction package (Donati et al. 1997).

Restricted to the wavelength ranges of $\mathrm{H} 13+\mathrm{H} 14$, the line list contains $48 \mathrm{Fe}$ I lines (92 initially). We checked the consistency of this reduced line list on Arcturus and the Sun but also on the 12 red clump stars observed with GIRAFFE and UVES. The same parameters were found for Arcturus with the reduced and total line lists: $T_{\text {eff }}=4300 \mathrm{~K}, \log g=2.5 \mathrm{dex}, \xi=1.5 \mathrm{~km} \mathrm{~s}^{-1}$, and $[\mathrm{Fe} / \mathrm{H}]=-0.52$ dex. For the Sun, we obtained $\log g=4.4$ dex, $\xi=1.5 \mathrm{~km} \mathrm{~s}^{-1}$, but $T_{\text {eff }}=5877 \mathrm{~K}$, i.e., increased by $100 \mathrm{~K}$ to fullfil the excitation equilibrium criterium. However, this has no impact on the deduced metallicity. For the 12 red clump stars, a new determination of the stellar parameters was performed with the reduced line list from the EWs measured on the UVES spectra with the same method as described in Lecureur et al. (2007). On average, the difference in the $T_{\text {eff }}$ values are nought with a dispersion of $100 \mathrm{~K}$, lower than the uncertainties. The $\xi$ resulting from the use of the reduced line list are slightly lower $\left(0.1 \mathrm{~km} \mathrm{~s}^{-1}\right)$ than the ones found with the total line list, which translates into $[\mathrm{Fe} / \mathrm{H}]$ values higher $(0.1 \mathrm{dex})$ than the previous ones. However, these systematics are smaller than the uncertainties found on the individual values, so we conclude that the stellar parameters determination from the reduced line is reliable.

\subsubsection{The microturbulence velocity determination}

In Lecureur et al. (2007), $\xi$ was determined so that lines of different observed EWs give the same iron abundance. However, the observed EW values are sensitive to uncertainties owing to either the quality of the spectra $(S / N$, resolving power) or the analysis method (continuum placement, FWHM fittings, etc.) which translates into correlated uncertainties on the corresponding $[\mathrm{Fe} \mathrm{I} / \mathrm{H}]$. This correlation between $[\mathrm{Fe} \mathrm{I} / \mathrm{H}]$ and observed EWs uncertainties leads to a bias towards higher $\xi$ as mentioned by Magain (1984).

To estimate this effect on the GIRAFFE sample, we simulated the observed EWs and measured $\xi$ for each simulation. This procedure was applied to the star $\mathrm{BWc}-2$ (a star representing our sample well: $T_{\text {eff }}=4656 \mathrm{~K}, \log g=2.30 \mathrm{dex}$,

\footnotetext{
3 Available: http://physics.nist.gov/asd3
}
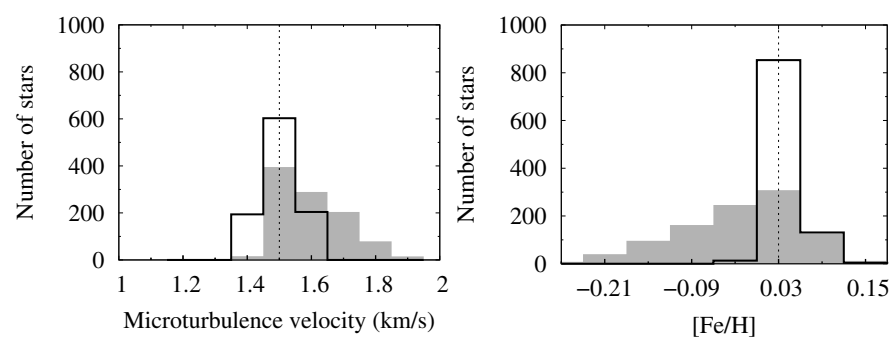

Fig. 3. Results of the simulations for the star BWc-2 ( $T_{\text {eff }}=4656 \mathrm{~K}$, $\log g=2.30 \mathrm{dex}, \xi=1.5 \mathrm{~km} \mathrm{~s}^{-1}$ and $\left.[\mathrm{Fe} \mathrm{I} / \mathrm{H}]=0.03\right)$. Left panel: histogram of $\xi$ values found with the observed EWs (shaded histogram) and theoretical EWs (black line). Right panel: histogram of the corresponding mean $[\mathrm{Fe} \mathrm{I} / \mathrm{H}]$ abundances.

$\xi=1.5 \mathrm{~km} \mathrm{~s}^{-1}$, and $\left.[\mathrm{Fe} \mathrm{I} / \mathrm{H}]=0.03\right)$. We simulated 1000 stars by randomly drawing a set of Fe $\mathrm{I}$ lines EWs $\left(\mathrm{EWs}_{\text {rand }}\right)$ around the expected EWs $\left(\mathrm{EWs}_{\mathrm{exp}}\right)$ with a standard deviation $\sigma=\delta_{\mathrm{EWs}}$ (given by DAOSPEC). For each 1000 simulated stars, Fe I abundances $\left([\mathrm{Fe} \mathrm{I} / \mathrm{H}]_{\text {rand }}\right)$ and corresponding uncertainties were computed from the random EWs using the stellar atmosphere model adopted for BWc-2. And $\xi$ was modified to minimise the slope $p$ of $[\mathrm{Fe} \mathrm{I} / \mathrm{H}]_{\text {rand }}$ versus $\mathrm{EWs}_{\text {rand }}$ such that $|p|<\sigma_{p}$, with $\sigma_{p}$ the uncertainty on $p$. The simulation results are shown in Fig. 3. The systematic effect on the determination of $\xi$ using the observed EWs is clearly illustrated by the $\xi$ distribution histogram. On average, $\xi$ has to be increased by $0.10 \pm 0.11 \mathrm{~km} \mathrm{~s}^{-1}$ leading to a corresponding systematic effect on the mean $[\mathrm{Fe} \mathrm{I} / \mathrm{H}]$ abundance, which decreases by $0.06 \pm 0.08$ dex.

This systematic effect can be suppressed using an abscissa linked to the observed EW but not affected by random errors. Magain (1984) suggests using the expected EWs (computed from the adopted stellar atmophere model and some assumed values of $\xi$ ) and proposed a scheme where the slope of $[\mathrm{Fe} \mathrm{I} / \mathrm{H}]$ versus $\mathrm{EWs}_{\text {exp }}$ is minimised to deduce $\xi$. Such $\xi$ values are then free of bias EW abundance error correlations. This scheme was applied to the previously simulated sample. As clearly illustrated by Fig. 3, the systematic effect on $\xi$ is no longer significant anymore: the mean $\xi$ value is of $1.50 \pm 0.06 \mathrm{~km} \mathrm{~s}^{-1}$ and the corresponding mean $[\mathrm{Fe} \mathrm{I} / \mathrm{H}]$ value is of $0.02 \pm 0.03 \mathrm{dex}$.

However, this method can introduce another type of bias on $\xi$ (and thus on $[\mathrm{Fe} / \mathrm{H}]$ ) if the $\mathrm{EWs}_{\text {exp }}$ are computed from an atmosphere model that is far from reality. In particular, $[\mathrm{Fe} / \mathrm{H}]$ needs to be known to $\sim 0.07$ dex. For this reason, $[\mathrm{Fe} / \mathrm{H}]$ was estimated as well as possible before $\xi$ was finally constrained.

\subsection{Final stellar parameters determination}

Starting with the photometric temperature, the final $T_{\text {eff }}$ was determined iteratively by requiring the excitation equilibrium on $\mathrm{Fe}_{\mathrm{I}}$ lines (no variation in $[\mathrm{Fe} \mathrm{I} / \mathrm{H}]$ with the excitation potential of the line $\chi_{\mathrm{ex}}$ ), and $\xi$ was constrained requiring no trend in the $\mathrm{Fe}_{\mathrm{I}}$ abundance as a function of the expected EW of the lines. At each step of the iteration on $\xi$, we checked that the metallicity of the atmophere model was the same as the one derived from the average Fe I abundance. As explained in Sect. 3.1, the photometric gravity was adopted as a final value and recomputed at each step of the iteration taking the change in $T_{\text {eff }}$ and $[\mathrm{Fe} / \mathrm{H}]$ into account. Stellar parameters and $[\mathrm{Fe} / \mathrm{H}]$ values were finally obtained for 219 red clump stars of the initial sample (228 stars), and are reported in Table 2, available at the CDS. The first lines of the table are reproduced in the printed version. 
Table 2. Adopted stellar parameters and measured $[\mathrm{Fe} / \mathrm{H}]$ and $[\mathrm{Mg} / \mathrm{H}]$ in the sample stars.

\begin{tabular}{lccccccccc}
\hline \hline ID & $\begin{array}{c}T_{\text {eff }} \\
\mathrm{K}\end{array}$ & $\begin{array}{c}\log g \\
\text { dex }\end{array}$ & $\begin{array}{c}\xi \\
\mathrm{kms}^{-1}\end{array}$ & $\begin{array}{c}{[\mathrm{Fe} / \mathrm{H}]} \\
\mathrm{dex}\end{array}$ & $\begin{array}{c}\sigma_{\mathrm{Fe}}(\text { low }) \\
\text { dex }\end{array}$ & $\begin{array}{c}\sigma_{\mathrm{Fe}}(\text { up }) \\
\text { dex }\end{array}$ & $\begin{array}{c}{[\mathrm{Mg} / \mathrm{H}]} \\
\text { dex }\end{array}$ & $\begin{array}{c}\sigma_{\mathrm{Mg}}(\mathrm{low}) \\
\text { dex }\end{array}$ & $\begin{array}{c}\sigma_{\mathrm{Mg}}(\text { up }) \\
\text { dex }\end{array}$ \\
\hline BWc-1 & 4528 & 2.09 & 1.4 & 0.29 & 0.14 & 0.13 & 0.34 & 0.08 & 0.08 \\
BWc-2 & 4556 & 2.25 & 1.4 & 0.04 & 0.10 & 0.21 & 0.29 & 0.10 & 0.10 \\
BWc-3 & 4713 & 2.23 & 1.5 & 0.33 & 0.17 & 0.28 & 0.42 & 0.15 & 0.15 \\
BWc-4 & 4912 & 2.28 & 1.4 & 0.01 & 0.10 & 0.19 & 0.26 & 0.10 & 0.10 \\
BWc-5 & 4636 & 2.21 & 1.5 & 0.58 & 0.12 & 0.33 & 0.63 & 0.14 & 0.14 \\
BWc-6 & 4769 & 2.19 & 1.2 & -0.17 & 0.17 & 0.30 & -0.00 & 0.19 & 0.19 \\
BWc-7 & 4769 & 2.19 & 1.2 & -0.17 & 0.17 & 0.30 & -0.00 & 0.19 & 0.19 \\
BWc-8 & 4810 & 2.31 & 1.4 & 0.49 & 0.15 & 0.17 & 0.47 & 0.09 & 0.09 \\
BWc-9 & 4685 & 2.21 & 1.5 & 0.17 & 0.12 & 0.24 & 0.21 & 0.12 & 0.12 \\
BWc-10 & 4690 & 2.13 & 1.5 & -0.13 & 0.14 & 0.24 & 0.20 & 0.11 & 0.11 \\
$\ldots$ & $\ldots$ & $\ldots$ & $\ldots$ & $\ldots$ & $\ldots$ & $\ldots$ & $\ldots$ & $\ldots$ & $\ldots$ \\
\hline
\end{tabular}

Notes. Full table is available at the CDS.

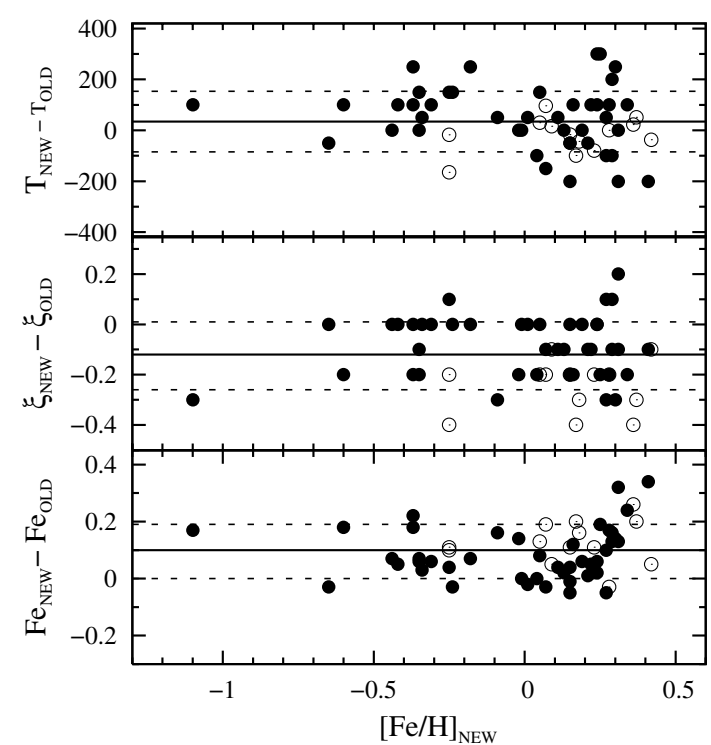

Fig. 4. Comparison of the stellar parameters $\left([\mathrm{Fe} / \mathrm{H}], \xi\right.$ and $\left.T_{\text {eff }}\right)$ obtained with the new $\xi$ determination method (NEW) and those published in Lecureur et al. (2007) (OLD). The black dots represent the RGB stars and the open circle the red clump stars. The black line shows the mean difference and the dotted lines the $\pm 1 \sigma$ dispersion.

This method was also applied to the UVES sample of Lecureur et al. (2007), using their full line list, leading to new stellar parameters for each star of the sample. Figure 4 shows the comparison between the new stellar parameters obtained with the new $\xi$ determination method and the "old" ones. There is no significant change in $T_{\text {eff }}$ values, the difference between old and new values is of $-34 \pm 119 \mathrm{~K}$. As expected with this new method, $\xi$ values are found systematically lower by $0.12 \pm 0.14 \mathrm{~km} \mathrm{~s}^{-1}$ on average, which translates into $[\mathrm{Fe} / \mathrm{H}]$ values systematically higher of $0.10 \pm 0.09$ dex. Compared to the whole sample, the red clump stars are more sensitive to the change in $\xi$ determination with a mean difference between old and new values that reaches $0.25 \mathrm{~km} \mathrm{~s}^{-1}$. This can be explained by lower $S / N$ for these stars compared to the total sample and consequently higher uncertainties on the EWs and corresponding Fe I abundances, making these stars more sensitive to the bias affecting the $\xi$ determination from the observed EWs.

\subsection{The stellar parameters uncertainties}

For each star of the red clump sample, an estimate of the uncertainty on $[\mathrm{Fe} / \mathrm{H}]$ has been computed from the uncertainties on the stellar parameters. The uncertainties on $T_{\mathrm{eff}}$ and $\xi$ have been evaluated from the uncertainties on the Fe I line EWs given by DAOSPEC, which translate into uncertainties in $\mathrm{Fe}$ I abundances derived from the EWs. These individual uncertainties on the Fe I lines were considered in the least squares line fit to the points in the $\left(\chi_{\text {ex }},[\mathrm{Fe} \mathrm{I} / \mathrm{H}]\right)$ plane to fix $T_{\text {eff }}$ and in the $(\mathrm{EWs},[\mathrm{Fe} \mathrm{I} / \mathrm{H}])$ plane to fix $\xi$ and are contained in the error on the line slope.

Keeping a fixed $\xi, T_{\text {eff }}$ upper and lower allowed boundaries were estimated by letting the slope $p$ reach its $1 \sigma$ uncertainty: $p \pm \sigma_{p}$. The thus determined boundaries are called $T_{\text {eff,up }}$ and $T_{\text {eff,lo }}$ and the corresponding uncertainties on $T_{\text {eff }}$ $\sigma_{\text {up }}\left(T_{\text {eff }}\right)$ and $\sigma_{\text {lo }}\left(T_{\text {eff }}\right)$. These temperature uncertainties need not be symmetric; in fact, for the 219 red clump stars, we found $\sigma_{\text {up }}\left(T_{\text {eff }}\right)=180 \pm 90 \mathrm{~K}$ and $\sigma_{\text {lo }}\left(T_{\text {eff }}\right)=220 \pm 90 \mathrm{~K}$, and half of the sample show asymmetric uncertainties on $T_{\text {eff }}$ that can reach $200 \mathrm{~K}$. Lower $\left(\sigma_{\mathrm{lo}}(\xi)\right)$ and upper $\left(\sigma_{\mathrm{up}}(\xi)\right)$ uncertainty values on $\xi$ have been estimated with the same principle fixing the $T_{\text {eff }}$ value. Unlike the $T_{\text {eff' }}$, these uncertainties are quasi symmetric with $\left|\sigma_{\mathrm{lo}}(\xi)\right|=\left|\sigma_{\text {up }}(\xi)\right|=0.21 \pm 0.08 \mathrm{~km} \mathrm{~s}^{-1}$.

A modification in $T_{\text {eff }}$ (or in $\xi$ ) value induces a change in $[\mathrm{Fe} / \mathrm{H}]$, which varies from star to star depending on the initial stellar parameters but also on the quality of the EW measurement ( $S / N$ ratio, continuum placement, etc.). As illustrated by Fig. 5, changing $T_{\text {eff }}$ by $200 \mathrm{~K}$ implies a change in $[\mathrm{Fe} / \mathrm{H}]$ around 0.10 dex for the coolest and more metallic stars of the sample and around 0.20 dex for the hotter and less metallic stars. Changes in $\xi$ by $0.2 \mathrm{~km} \mathrm{~s}^{-1}$ lead to similar variations in the $[\mathrm{Fe} / \mathrm{H}]$ value: around 0.10 dex for the coolest and more metallic stars of the sample and around 0.16 dex for the hotter and less metallic stars. Overall in our 219 clump star sample, the uncertainties on $[\mathrm{Fe} / \mathrm{H}]$ associated to $T_{\text {eff }}$ uncertainties are $0.15 \pm 0.07$ dex on average, and those associated to $\xi$ uncertainties, of $0.16 \pm 0.06$ dex. The individual values are reported in Table 3 , available at the CDS.

The uncertainty associated with $[\mathrm{Fe} / \mathrm{H}]$ were computed with the formalism described in McWilliam et al. (1995) taking the errors into account on the model atmosphere stellar parameters $T_{\text {eff }}$ and $\xi$ and the errors on the Fe I line EW measurements. Errors on $[\mathrm{Fe} / \mathrm{H}]$ coming from the $\log g$ value or the metallicity adopted to compute the stellar atmosphere model were found to be negligible compared to the other sources. Finally, the variance on $[\mathrm{Fe} / \mathrm{H}], \sigma^{2}(\mathrm{Fe})$, can be written as

$$
\begin{aligned}
\sigma^{2}(\mathrm{Fe})= & \left(\frac{\partial \epsilon}{\partial W}\right)^{2} \sigma_{W}^{2}+\left(\frac{\partial \epsilon}{\partial T}\right)^{2} \sigma_{T}^{2}+\left(\frac{\partial \epsilon}{\partial \xi}\right)^{2} \sigma_{\xi}^{2} \\
& +2\left(\frac{\partial \epsilon}{\partial T}\right)\left(\frac{\partial \epsilon}{\partial \xi}\right) \sigma_{T, \xi},
\end{aligned}
$$


Table 3. Errors in stellar parameters and associated errors on Fe for the sample stars.

\begin{tabular}{|c|c|c|c|c|c|c|c|c|c|c|c|c|}
\hline \multirow[b]{2}{*}{ ID } & \multirow[b]{2}{*}{$\begin{array}{c}T_{\text {eff }} \\
\mathrm{K}\end{array}$} & \multirow[b]{2}{*}{$\begin{array}{l}\log g \\
\operatorname{dex}\end{array}$} & \multirow[b]{2}{*}{$\begin{array}{c}\xi \\
\mathrm{kms}^{-1} \\
\end{array}$} & \multirow[b]{2}{*}{$\begin{array}{c}{[\mathrm{Fe} / \mathrm{H}]} \\
\operatorname{dex}\end{array}$} & \multicolumn{4}{|c|}{ Upper } & \multicolumn{4}{|c|}{ Lower } \\
\hline & & & & & $\begin{array}{c}\sigma_{\xi} \\
\mathrm{kms}^{-1}\end{array}$ & $\begin{array}{c}\sigma_{\xi}(\mathrm{Fe}) \\
\operatorname{dex}\end{array}$ & $\begin{array}{c}\sigma_{T} \\
\mathrm{~K}\end{array}$ & $\begin{array}{c}\sigma_{T}(\mathrm{Fe}) \\
\operatorname{dex}\end{array}$ & $\begin{array}{c}\sigma_{\xi} \\
\mathrm{kms}^{-1}\end{array}$ & $\begin{array}{c}\sigma_{\xi}(\mathrm{Fe}) \\
\operatorname{dex}\end{array}$ & $\begin{array}{c}\sigma_{T} \\
\mathrm{~K}\end{array}$ & $\begin{array}{c}\sigma_{T}(\mathrm{Fe}) \\
\quad \operatorname{dex}\end{array}$ \\
\hline$\overline{B W c-1}$ & 4528 & 2.09 & 1.4 & +0.29 & -0.10 & +0.07 & +150 & +0.10 & +0.20 & -0.16 & -200 & -0.10 \\
\hline BWc-2 & 4556 & 2.25 & 1.4 & +0.04 & -0.20 & +0.19 & +100 & +0.08 & +0.10 & -0.07 & -150 & -0.07 \\
\hline BWc-3 & 4713 & 2.23 & 1.5 & +0.33 & -0.20 & +0.16 & +300 & +0.23 & +0.30 & -0.22 & -50 & -0.03 \\
\hline BWc-4 & 4912 & 2.28 & 1.4 & +0.01 & -0.20 & +0.19 & +100 & +0.10 & +0.10 & -0.06 & -100 & -0.09 \\
\hline BWc-5 & 4636 & 2.21 & 1.5 & +0.58 & -0.30 & +0.30 & +300 & +0.17 & +0.10 & -0.12 & -400 & -0.16 \\
\hline BWc-6 & 4769 & 2.19 & 1.2 & -0.17 & -0.20 & +0.15 & +300 & +0.26 & +0.20 & -0.13 & -200 & -0.15 \\
\hline BWc-7 & 4769 & 2.19 & 1.2 & -0.17 & -0.20 & +0.15 & +300 & +0.26 & +0.20 & -0.13 & -200 & -0.15 \\
\hline BWc-8 & 4810 & 2.31 & 1.4 & +0.49 & -0.10 & +0.10 & +150 & +0.13 & +0.10 & -0.07 & -200 & -0.14 \\
\hline BWc-9 & 4685 & 2.21 & 1.5 & +0.17 & -0.20 & +0.20 & +150 & +0.13 & +0.20 & -0.14 & -200 & -0.15 \\
\hline BWc-10 & 4690 & 2.13 & 1.5 & -0.13 & -0.20 & +0.15 & +200 & +0.18 & +0.30 & -0.21 & -200 & -0.14 \\
\hline$\ldots$ & $\ldots$ & $\ldots$ & $\ldots$ & $\ldots$ & $\ldots$ & $\ldots$ & $\ldots$ & $\ldots$ & $\ldots$ & $\ldots$ & $\ldots$ & $\ldots$ \\
\hline
\end{tabular}

Notes. Full table is available at the CDS.
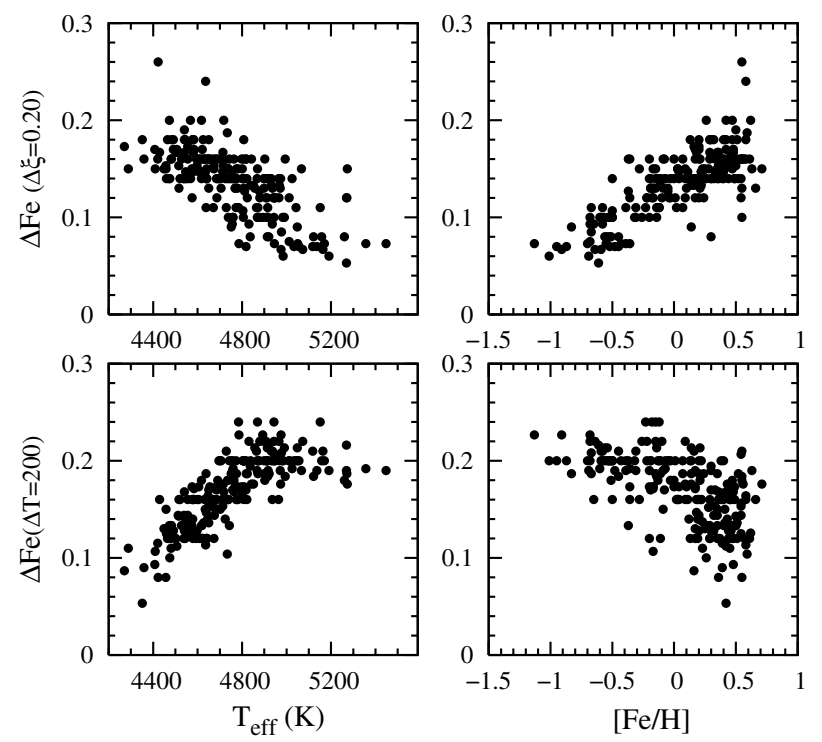

Fig. 5. Variation in the resulting $[\mathrm{Fe} / \mathrm{H}]$ with increasing $T_{\text {eff }}$ by $200 \mathrm{~K}$ (lower panels) and $\xi$ by $0.2 \mathrm{~km} \mathrm{~s}^{-1}$ (upper panels) as a function of the initial $T_{\text {eff }}$ (left panels) and of the initial $[\mathrm{Fe} / \mathrm{H}]$ value (right panels). The hotter and less metallic stars are the most sensitive to a modification of $T_{\text {eff }}$ whereas the cooler and more metallic stars are the most sensitive to a modification of $\xi$.

with $\epsilon$ the $\mathrm{Fe}$ abundance, $\sigma_{\mathrm{W}}^{2}, \sigma_{T}^{2}$ and $\sigma_{\xi}^{2}$ the variance in the EWs measurement, $T_{\mathrm{eff}}$ and $\xi$, respectively, and $\sigma_{\mathrm{T}, \xi}$ the covariance between $T_{\text {eff }}$ and $\xi$. The covariances between the EW measurements and the stellar parameters $\xi$ or $T_{\text {eff }}$ are nought because these variables are independent. The terms $\left(\frac{\partial \epsilon}{\partial T}\right)^{2} \sigma_{T}^{2}$ and $\left(\frac{\partial \epsilon}{\partial \xi}\right)^{2} \sigma_{\xi}^{2}$ were computed as explained in the previous paragraph. The variance in the EW measurements and the associated uncertainty on $[\mathrm{Fe} / \mathrm{H}]$ were estimated from the line-to-line dispersion divided by the number of lines. The covariance between $T_{\text {eff }}$ and $\xi$ were approximated by measuring the variation in the slope fixing $T_{\text {eff }}$ (resp. $\xi$ ) due to changes in $\xi$ (resp. $T_{\text {eff }}$ ).

The total upper and lower uncertainty values on $[\mathrm{Fe} / \mathrm{H}]$ and $[\mathrm{Mg} / \mathrm{H}]$ computed from Eq. (2) are reported in Table 2, available at the CDS, while Table 3 details the different contributions of the stellar parameter uncertainties $\left(\sigma_{\xi}\right.$ and $\left.\sigma_{T}\right)$ propagated onto the abundances $\left(\sigma_{\xi}(\mathrm{Fe})\right.$ and $\left.\sigma_{T}(\mathrm{Fe})\right)$. For the total red clump sample (219 stars), and as illustrated in Fig. 6, the lower values range from 0.04 to 0.35 dex with a median value of $0.17 \pm 0.05$ and show no trend with metallicity. The upper values
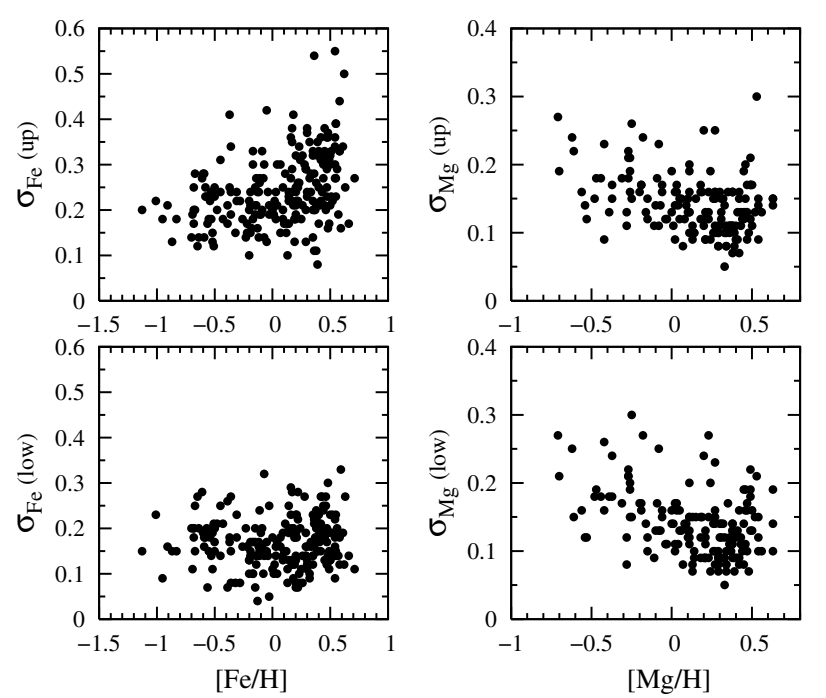

Fig. 6. Left panels: upper and lower errors on $[\mathrm{Fe} / \mathrm{H}]$ as a function of $[\mathrm{Fe} / \mathrm{H}]$ for the 219 red clump stars. The upper values increase with the metallicity with mean values around 0.3 dex for the most metallic stars. The lower values show no trend with $[\mathrm{Fe} / \mathrm{H}]$ with a median value of 0.17 dex for the whole sample. Right panels: upper and lower errors on $[\mathrm{Mg} / \mathrm{H}]$ as a function of $[\mathrm{Mg} / \mathrm{H}]$ for the 162 red clump stars selected from the total sample. At low $[\mathrm{Mg} / \mathrm{H}]$, the abundance errors are dominated by the line measurement rather than stellar parameter uncertainties because the $\mathrm{Mg}$ triplet lines become very weak.

are systematically higher with a median value of $0.24 \pm 0.08$ and show an increase with the metallicity reaching 0.5 dex for supersolar metallicity stars.

We also mention that a mean error in $[\mathrm{Fe} / \mathrm{H}]$ was also estimated by Zoccali et al. (2008) by comparing the measured metallicities in the 50 stars (RGB and red clump stars of the Lecureur et al. 2007, sample) observed both with GIRAFFE and UVES. They find no systematic offset on the $[\mathrm{Fe} / \mathrm{H}]$ values with a scatter of $0.16 \mathrm{dex}$, a value in agreement with the previous estimates from the total GIRAFFE red clump sample.

\subsection{Magnesium abundances}

The Mg abundances were determined from the $6319 \AA$ triplet using spectral synthesis fitting. The synthetic spectra were computed using the LTE spectral analysis code "turbospectrum" (described in Alvarez \& Plez 1998) and the molecular and atomic line lists described in Lecureur et al. (2007). In short, we recall 

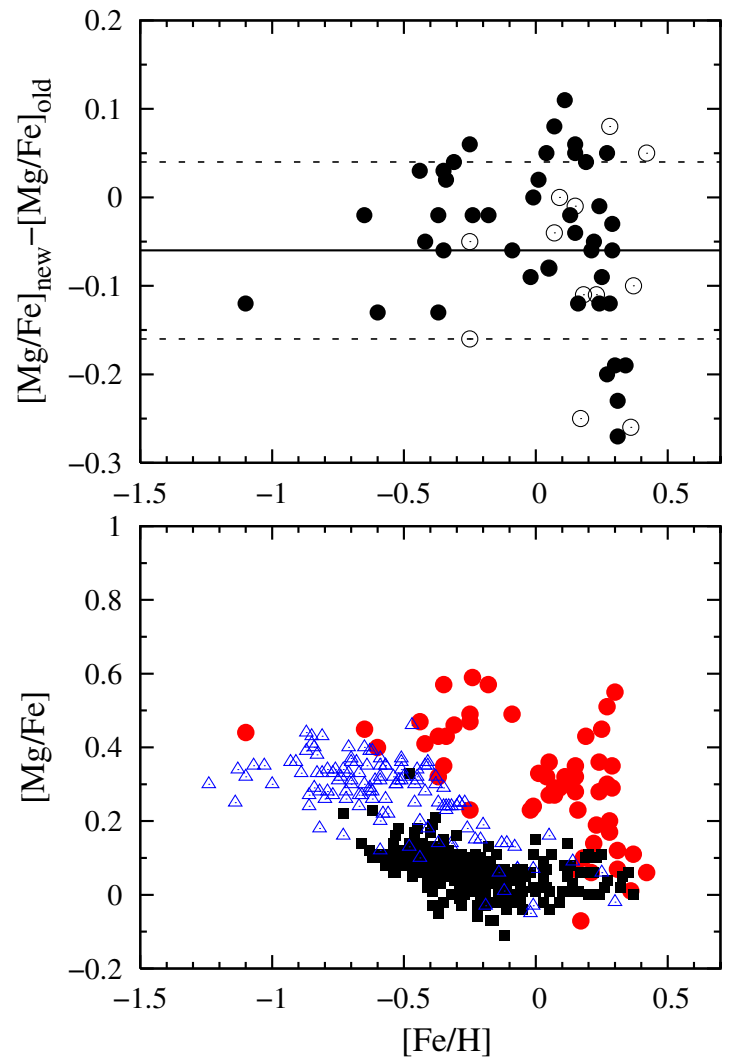

Fig. 7. Upper panel: difference in $[\mathrm{Mg} / \mathrm{Fe}]$ found for the UVES red clump (open circle) and RGB stars (full circle) with the old and new sets of parameters as a function of the new metallicities. The black line shows the mean value of this difference $(-0.06)$ and the two dotted lines the $\pm 1 \sigma$ dispersion $(\sigma=0.10)$. Lower panel: $[\mathrm{Mg} / \mathrm{Fe}]$ in the UVES bulge stars (red points, computed with the new stellar parameters) compared with the $[\mathrm{Mg} / \mathrm{Fe}]$ in the thin (black squares) and thick (blue triangles) discs stars from Bensby et al. (2004, 2005), Reddy et al. (2006).

that the atomic line list is the one of the VALD database (Kupka et al. 1999, and references therein) with (i) solar astrophysical $\log g f$-values for the three lines of the $\mathrm{Mg}$ triplet and (ii) the $\lambda$ $6318.1 \AA \mathrm{Ca}$ I autoionisation line broadening adjusted in order to reproduce the CaI dip in the Sun, Arcturus, and $\mu$ Leo. The molecular line lists included in our syntheses are the following: $\mathrm{C}_{2}\left({ }^{12} \mathrm{C}^{12} \mathrm{C}\right.$ and $\left.{ }^{12} \mathrm{C}^{13} \mathrm{C}\right)$ Swan system $(\mathrm{A}-\mathrm{X}), \mathrm{CN}\left({ }^{12} \mathrm{C}^{14} \mathrm{~N}\right.$ and $\left.{ }^{13} \mathrm{C}^{14} \mathrm{~N}\right)$ red system $(\mathrm{A}-\mathrm{X}), \mathrm{TiO} \gamma$ and $\gamma^{\prime}$ systems Plez (1998). In and near the triplet, the $\log g f$ values of some $\mathrm{CN}$ lines were modified to obtain the same $\mathrm{Mg}$ abundance from the three lines of the triplet in the three reference stars.

\subsubsection{In the UVES stars: new determination}

Adopting the new stellar parameters and metallicities (see Sect. 3.3), the Mg abundances were determined for the UVES stars as described in Lecureur et al. (2007) with a $\mathrm{Ca}$ abundance computed from the following relation: $[\mathrm{Ca} / \mathrm{Fe}]=-0.20[\mathrm{Fe} / \mathrm{H}]+$ 0.06. This linear relation was obtained on the UVES sample by a least square fit to the $[\mathrm{Ca} / \mathrm{Fe}]$ values deduced from EWs.

The mean difference in $[\mathrm{Mg} / \mathrm{H}]$ between the two determinations is very small (as expected because $[\mathrm{Mg} / \mathrm{H}]$ is not sensitive to a change of $\xi):[\mathrm{Mg} / \mathrm{H}]_{\text {new }}-[\mathrm{Mg} / \mathrm{H}]_{\text {old }}=0.03 \pm 0.06 \mathrm{dex}$ but becomes more significant on $[\mathrm{Mg} / \mathrm{Fe}]$ with $[\mathrm{Mg} / \mathrm{Fe}]_{\text {new }}-$ $[\mathrm{Mg} / \mathrm{Fe}]_{\text {old }}=-0.06 \pm 0.10 \mathrm{dex}$ (see Fig. 7). However, the global trend of $[\mathrm{Mg} / \mathrm{Fe}]$ vs. $[\mathrm{Fe} / \mathrm{H}]$ is almost not affected by this difference, as illustrated by Fig. 7, which shows the new $[\mathrm{Mg} / \mathrm{Fe}]$ in the bulge stars and the $[\mathrm{Mg} / \mathrm{Fe}]$ found in the thin and thick discs stars by Bensby et al. $(2004,2005)$ and Reddy et al. (2006). In the lower metallicity range $([\mathrm{Fe} / \mathrm{H}]<-0.4)$, the bulge stars are not as separated from the thick disc stars as they were in (Lecureur et al. 2007, their Fig. 6) with $[\mathrm{Mg} / \mathrm{Fe}]$ values in bulge stars similar to the highest values found in the thick disc stars. For $-0.4 \lesssim[\mathrm{Fe} / \mathrm{H}] \lesssim 0.1$, the bulge still shows higher $[\mathrm{Mg} / \mathrm{Fe}]$ values than those found in both discs. At higher metallicities, the dispersion has slightly decreased (mainly from our use of the linear relation to compute the $\mathrm{Ca}$ abundance rather than relying on individual - more uncertain - values for each star) but remains high, with $[\mathrm{Mg} / \mathrm{Fe}]$ values from thin disc values $([\mathrm{Mg} / \mathrm{Fe}] \sim 0.1)$ to 0.55 . The conclusions drawn by Lecureur et al. (2007) from the $[\mathrm{Mg} / \mathrm{Fe}]$ values are still valid with the $[\mathrm{Mg} / \mathrm{Fe}]$ computed from the new stellar parameters. The abundances of $\mathrm{O}, \mathrm{Al}$, and $\mathrm{Na}$ in the UVES stars will also be updated using these new stellar parameters and be published and discussed in a future paper.

\subsubsection{In the GIRAFFE stars}

From the total red clump sample (219 stars), we selected stars according to their uncertainties on the $[\mathrm{Fe} / \mathrm{H}]$ values in order to exclude stars for which $\mathrm{Mg}$ would be measured with uncertainties that are too large (mainly stars with low $S / N$ spectra). Only stars with $\sigma_{\text {up }}(\mathrm{Fe})<0.30$ were kept to provide a new subsample of 162 stars. To compute the synthetic spectrum, the abundances of $\mathrm{C}, \mathrm{N}, \mathrm{O}$, and $\mathrm{Ca}$ are needed for each star of the GIRAFFE sample. As a result, from the decrease in spectral resolution, the $\mathrm{C}, \mathrm{N}$, and $\mathrm{O}$ indicators measurable in the UVES spectra are either nondetectable in the GIRAFFE spectra or would lead to large uncertainties in the resulting abundances, mainly due to uncertainties in the continuum placement $(\sim 0.5$ dex on $[\mathrm{C} / \mathrm{H}]$ and $\sim 0.3 \mathrm{dex}$ on $[\mathrm{O} / \mathrm{H}])$. Therefore, the synthetic spectra were computed with the mean values, $[\mathrm{C} / \mathrm{Fe}]=-0.04$ and $[\mathrm{N} / \mathrm{Fe}]=+0.43$, found in the UVES star sample. The $\mathrm{O}$ abundance was deduced from the following linear relation: $[\mathrm{O} / \mathrm{Fe}]=-0.56[\mathrm{Fe} / \mathrm{H}]+0.22$ fitted by least squares to the $[\mathrm{O} / \mathrm{Fe}]$ values found for the UVES stars sample of Zoccali et al. (2006). The Ca abundance was computed as in Sect. 3.5.1.

Each observed spectrum was first normalised using the continuum found by DAOSPEC. The continuum was then adjusted by a visual inspection of a $10 \AA$ wavelength interval centred on the triplet. To well identify the possible absorption from the $\mathrm{CN}$ lines and/or from the $\mathrm{Ca}$ I line, two other synthetic spectra were overlaid: (i) one spectral synthesis only with the molecular lines and (ii) one spectral synthesis only with the Ca I. In $\mu$ Leo, both $\mathrm{CN}$ and $\mathrm{Ca}$ I affect the region, while in the more metal-poor star BWc-93, the contamination by the $\mathrm{Ca}$ I and CN lines is negligible. This visual analysis also permits flagging or rejecting some stars from the final $\mathrm{Mg}$ measurement for one of the following reasons: (i) presence of telluric lines affecting the $\mathrm{Mg}$ measurement; (ii) too low $S / N$ in the $\mathrm{Mg}$ region, which can affect the continuum placement; and (iii) strong disagreement between observed and synthetic spectra.

At GIRAFFE resolution, blends between the two redder lines of the triplet and between the Mg I line at $6318.72 \AA$ and the Fe I line at $6318.1 \AA$ become more important as illustrated by Fig. 8. This figure also shows that line list uncertainties do not have a significant impact on the $\mathrm{Mg}$ abundance determination from UVES spectra, but become larger at GIRAFFE resolution and can affect the $\mathrm{Mg}$ value. More specifically, in the synthetic spectra, the absorption around 6318.2 $\AA$ is overestimated and at 

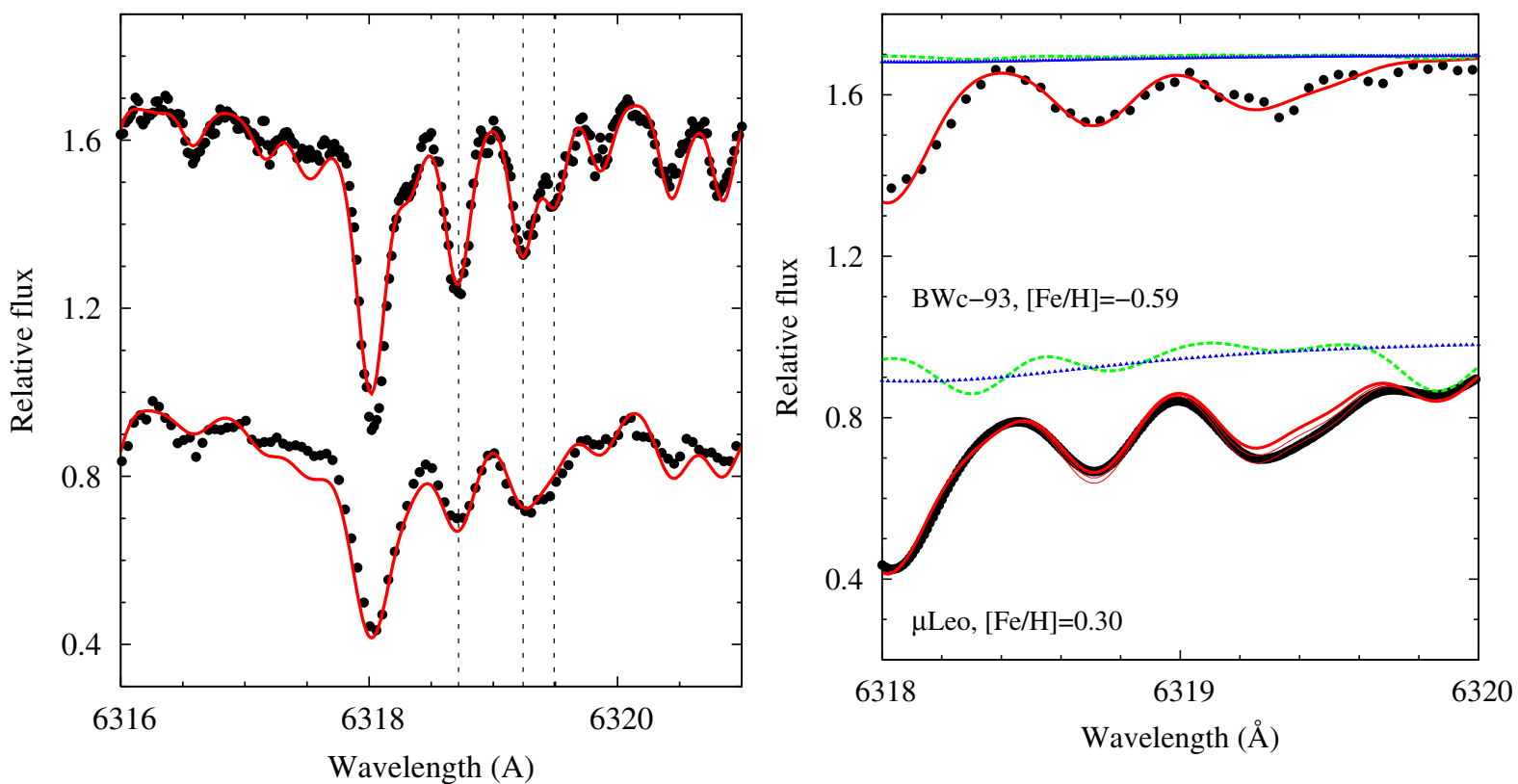

Fig. 8. Left panel: observed spectrum (black points) around the $\mathrm{Mg}$ I triplet for the BWc-1 red clump star obtained with GIRAFFE (lower spectrum) and UVES (upper spectrum). The red lines represent the synthetic spectra computed with $[\mathrm{Mg} / \mathrm{H}]=+0.34$ and $[\mathrm{Mg} / \mathrm{H}]=+0.38$ respectively for GIRAFFE and UVES convolution. Right panel: comparison of observed (black points) and synthetic spectra (red lines) computed with $[\mathrm{Mg} / \mathrm{H}]=-0.16$ for the red clump star BWc-93 and $[\mathrm{Mg} / \mathrm{H}]=+0.42,+0.32,+0.22$ for $\mu$ Leo. The blue dotted lines and the green thick dashed lines represent the absorption of the $\mathrm{Ca}$ I line and of the $\mathrm{CN}$ lines respectively.

GIRAFFE resolution, the left wing of the $\mathrm{Mg}$ I line at $6318.72 \AA$ become more contaminated leading to an underestimation of the $\mathrm{Mg}$ abundance deduced from this line. These effects were measured on $\mu$ Leo by comparing the observed spectrum convolved to the GIRAFFE resolution with the synthetic spectrum computed with the stellar parameters, $\mathrm{C}, \mathrm{N}, \mathrm{O}$ and $\mathrm{Ca}$ abundances for $\mu$ Leo from Lecureur et al. (2007) and different Mg abundances (see Fig. 8, right panel). The abundance deduced from the two redder lines of the triplet is $[\mathrm{Mg} / \mathrm{H}]=0.42$, whereas the abundance deduced from the line at $6318.72 \AA$ is 0.2 dex lower. On the other hand, in the more metal-poor star BWc-93 the three triplet lines yield the same abundance.

Due to the previous considerations, for each star of the subsample, two estimations of the $\mathrm{Mg}$ abundance were computed by minimising the $\chi^{2}$ values between normalised observed and synthetic spectra on a wavelength domain restricted (i) to the region covered by the two redder lines $\left([\mathrm{Mg} / \mathrm{H}]_{2 \mathrm{Lines}}\right)$ and (ii) to the region covered by the three lines of the $\mathrm{Mg}$ triplet $\left([\mathrm{Mg} / \mathrm{H}]_{\text {triplet }}\right)$. For the total sample, the $\mathrm{Mg}$ abundance deduced from the triplet is 0.05 dex lower on average than the one deduced from the two redder lines with a dispersion of 0.06 around the mean. For the less metallic stars, the difference $[\mathrm{Mg} / \mathrm{H}]_{2 \text { Lines }}-[\mathrm{Mg} / \mathrm{H}]_{\text {triplet }}$ can be negative and the mean difference is nought. In this metallicity range, the triplet absorption weakens and the abundance deduced from the two redder lines becomes more sensitive to uncertainties $(S / N$, continuum placement). For stars with $[\mathrm{Fe} / \mathrm{H}]>$ -0.4 dex, the difference starts to be positive and increases with the metallicity of the star to reach 0.15 dex for the more metallic stars. Moreover, at supersolar metallicities, the $[\mathrm{Mg} / \mathrm{Fe}]$ values deduced from the triplet are more dispersed than those deduced from the two redder lines. In our red clump GIRAFFE sample, to allow for both these considerations, we finally adopted the $[\mathrm{Mg} / \mathrm{H}]$ value deduced from the triplet for the stars with $[\mathrm{Fe} / \mathrm{H}]<-0.4$ dex and the value deduced from the two redder lines of the triplet for the stars with $[\mathrm{Fe} / \mathrm{H}]>-0.4$ dex.

To evaluate the $\mathrm{Mg}$ determination for the red clump stars, we compared the $\mathrm{Mg}$ abundances found from the GIRAFFE spectra $\left([\mathrm{Mg} / \mathrm{H}]_{\mathrm{GIR}}\right)$ with those found from the UVES spectra $\left([\mathrm{Mg} / \mathrm{H}]_{\text {UVES }}\right.$ ) for the stars observed with both instruments (see Fig. 9). To increase the statistics of the comparison, we also computed with the same method the Mg abundances in 30 RGB stars of the Lecureur et al. (2007) sample from the GIRAFFE spectra with the stellar parameters determined by Zoccali et al. (2008). For the total sample (44 stars), a systematic difference between the two determinations is found: $[\mathrm{Mg} / \mathrm{H}]_{\mathrm{GIR}}-[\mathrm{Mg} / \mathrm{H}]_{\mathrm{UVES}}=$ $-0.06 \pm 0.16 \mathrm{dex}$, which does not depend on the metallicity. This difference is small, and can be mainly explained by the resolution decrease that affects the $\mathrm{Mg}$ determination via uncertainties in the continuum placement and/or by uncertainties in the atomic and molecular line list that affect the line fitting differently at different resolutions. Both red clump and RGB stars show the same behaviour.

However, in terms of comparison with abundances found in the discs, this small difference can become significant. Figure 9 shows the $[\mathrm{Mg} / \mathrm{Fe}]$ ratios found from GIRAFFE and UVES spectra of the same stars. The two sets of points show a similar global trend, although for $-0.3<[\mathrm{Fe} / \mathrm{H}]<0,[\mathrm{Mg} / \mathrm{Fe}]_{\mathrm{GIR}}$ ratios are closer to those found in thick discs stars at the same metallicity than the $[\mathrm{Mg} / \mathrm{Fe}]_{\text {UVES }}$ were. At higher metallicity $([\mathrm{Fe} / \mathrm{H}]>0.2)$, the dispersion on $[\mathrm{Mg} / \mathrm{Fe}]_{\mathrm{GIR}}$ values is considerably lower than that of $[\mathrm{Mg} / \mathrm{Fe}]_{\text {UvEs. }}$. We attribute the larger dispersion found from the UVES spectra to the result of the lower $S / N$ for these spectra. From both sets of measurements, the bulge stars have $[\mathrm{Mg} / \mathrm{Fe}]$ higher on average than those of the thin disc stars, but half of the stars fall within the thin disc trend from the GIRAFFE measurement, which was not the case from the UVES measurement. The $[\mathrm{Mg} / \mathrm{Fe}]$ trend will be further discussed in Sect. 4.1 for the total red clump sample, but we would like to note at this point that the previous sample of Lecureur et al. (2007) plotted here was a mix of red clump (13) in Baade's window and RGB stars (44) coming from four different fields located at Galactic latitudes of $-3^{\circ},-4^{\circ}$ (Baade's Window), $-6^{\circ}$ to $-12^{\circ}$. Among these fields, some are expected to be more contaminated by foreground Galactic discs (thin and thick), and this 

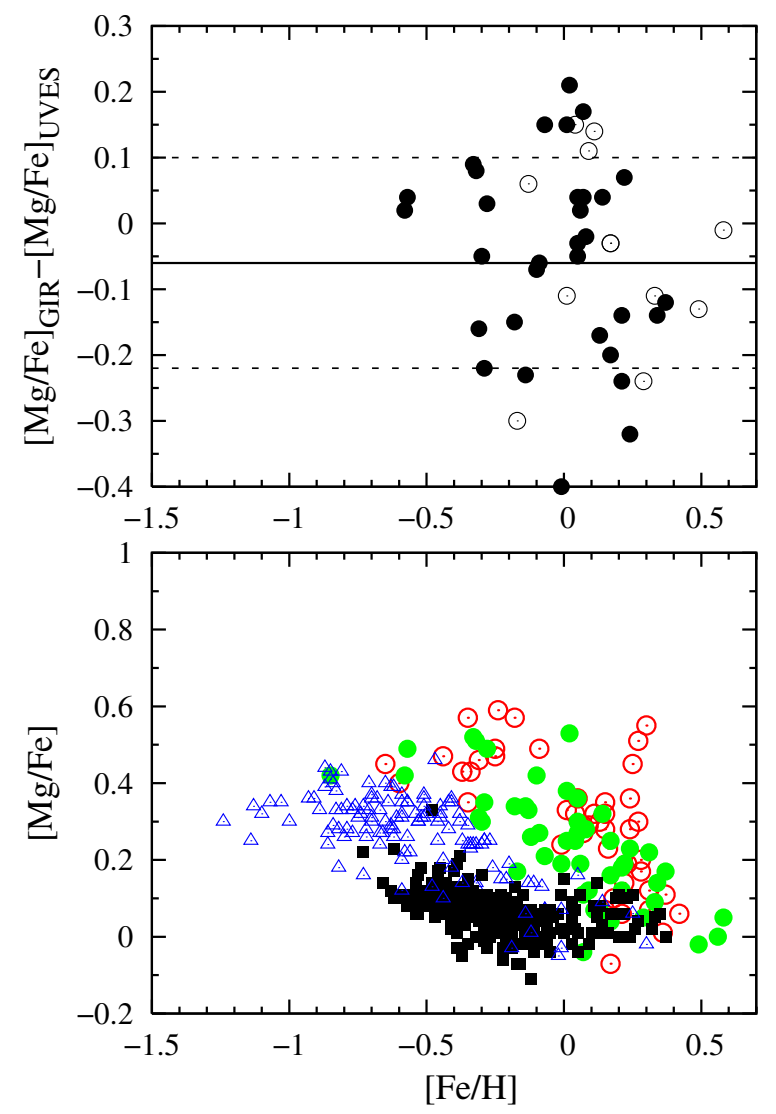

Fig. 9. Lower panel: comparison of the $[\mathrm{Mg} / \mathrm{Fe}]$ found from GIRAFFE spectra (green filled circles) with $[\mathrm{Mg} / \mathrm{Fe}]$ found from UVES spectra for 44 bulge stars of the Lecureur et al. (2007) sample (open dotted circles). The thin (black filled squares) and thick (blue open triangles) discs abundances come from Bensby et al. $(2004,2005)$ and Reddy et al. (2006). Upper panel: difference in $[\mathrm{Mg} / \mathrm{Fe}]$ found from GIRAFFE and UVES spectra for 44 stars (red clump open circle and RGB stars full circle) observed with both instruments. The black line shows the mean value of this difference $(-0.06)$ and the two dotted lines show the $\pm 1 \sigma$ around $(\sigma=0.16)$.

could partly explain the difference between the sample studied in Lecureur et al. (2007) and the pure Baade's window sample studied in the present paper.

\subsection{3. $[\mathrm{Mg} / \mathrm{H}]$ and $[\mathrm{Fe} / \mathrm{H}]$ uncertainties for the red clump sample}

The individual uncertainties on $[\mathrm{Mg} / \mathrm{H}]$ and $[\mathrm{Fe} / \mathrm{H}]$ were estimated from uncertainties associated with the $\chi^{2}$ procedure $\left(\sigma_{\text {FIT }}\right)$, as well as the uncertainties linked to uncertainties on stellar parameters ( $T_{\mathrm{eff}}$ and $\xi$ computed in Sect. 3.4). The value of $\sigma_{\text {FIT }}$ was computed using the $\delta \chi^{2}=1$ contour. The uncertainty on $[\mathrm{Mg} / \mathrm{H}]$ due to the uncertainty on $T_{\text {eff }}$ and on $\xi$ was computed with the following relations: $\sigma([\mathrm{Mg} / \mathrm{H}])_{T}=\left(\frac{\mathrm{d}[\mathrm{Mg} / \mathrm{H}]}{\mathrm{d} T}\right) \sigma(T)$ and $\sigma([\mathrm{Mg} / \mathrm{H}])_{\xi}=\left(\frac{\mathrm{d}[\mathrm{Mg} / \mathrm{H}]}{\mathrm{d} \xi}\right) \sigma(\xi)$ with $\sigma(T)$ and $\sigma(\xi)$ the uncertainties on $T_{\text {eff }}$ and $\xi$ respectively. Both $\left(\frac{\mathrm{d}[\mathrm{Mg} / \mathrm{H}]}{\mathrm{d} T}\right)$ and $\left(\frac{\mathrm{d}[\mathrm{Mg} / \mathrm{H}]}{\mathrm{d} \xi}\right)$ were estimated from the $[\mathrm{Mg} / \mathrm{H}]$ dependence on $T_{\text {eff }}$ and $\xi$ variations found in the UVES stars (see Table 10 of Lecureur et al. 2007). Errors arising from $\log \mathrm{g}$ uncertainties (an error of $0.3 \mathrm{dex}$ on $\log g$ corresponds to an error of 0.02 , on average) were found to be negligible compared to the other sources.

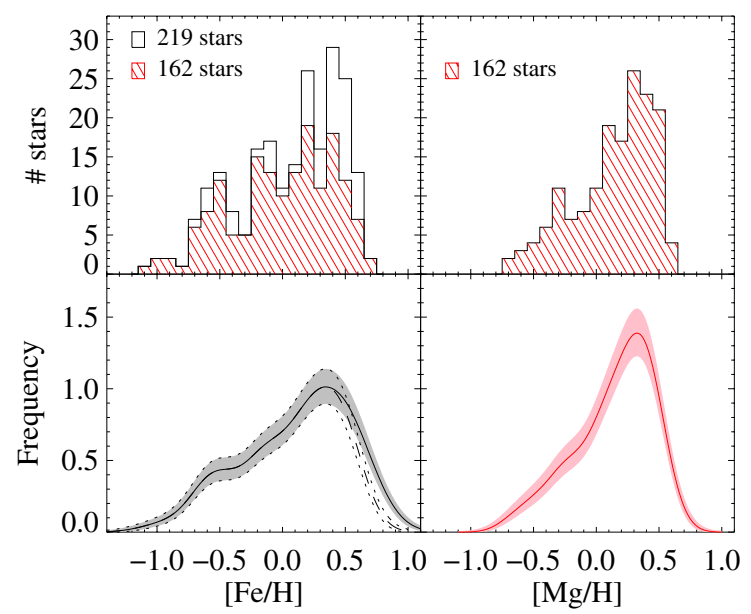

Fig. 10. Upper left panel: distribution of $[\mathrm{Fe} / \mathrm{H}]$ for the 219 bulge clump stars in Baade's window (thick histogram) and distribution of $[\mathrm{Fe} / \mathrm{H}]$ for the 162 star subsample for which $[\mathrm{Mg} / \mathrm{H}]$ is measured (hatched histogram). Upper right panel: distribution of $[\mathrm{Mg} / \mathrm{H}]$ for the 162 bulge clump stars in Baade's window. Lower left panel: smoothed distribution of $[\mathrm{Fe} / \mathrm{H}]$ derived from the upper panel, with its associated variability band. The initial metal-rich side of the distribution (dashed line) has been stretched (full line) to make it consistent with the error law and to achieve the deconvolution (see text). Lower right panel: smoothed distribution of $[\mathrm{Mg} / \mathrm{H}]$ derived from the upper panel, with its associated variability band (see text).

\section{Metallicity distributions}

In the following we discuss the resulting metallicity distribution (MD) for our sample, using in turn $\mathrm{Fe}$ and $\mathrm{Mg}$ as a proxy for metallicity. We first examine the raw MDs and their robustness, then proceed to analyse their underlying shape (taking errors into account) and finally examine the $[\mathrm{Mg} / \mathrm{Fe}]$ trend with metallicity in the sample.

\subsection{Raw metallicity distributions}

\subsection{1. $[\mathrm{Fe} / \mathrm{H}]$ metallicity distribution}

$\mathrm{The}[\mathrm{Fe} / \mathrm{H}] \mathrm{MD}$ for the 219 bulge red clump stars is shown in Fig. 10. The mean $[\mathrm{Fe} / \mathrm{H}]$ value is $+0.05 \pm 0.03 \mathrm{dex}$, and the full range of metallicities spans $-1.13<[\mathrm{Fe} / \mathrm{H}]<0.71 \mathrm{dex}$. The distribution is clearly asymmetric with a median $[\mathrm{Fe} / \mathrm{H}]$ of 0.19 dex, and shows a high proportion of metal-rich stars: $65 \%$ with supersolar metallicities and $25 \%$ with $[\mathrm{Fe} / \mathrm{H}]>0.38$ dex. For $[\mathrm{Fe} / \mathrm{H}]>0.50 \mathrm{dex}$, the number of stars decreases considerably.

The MD shows two low-level secondary peaks around $[\mathrm{Fe} / \mathrm{H}]=-0.55$ and around $[\mathrm{Fe} / \mathrm{H}]=-0.20$ dex, both with very low statistics. While the first remains regardless of the size and centre of the histogram bins, the second results from a small accumulation of stars in a very narrow metallicity range around $[\mathrm{Fe} / \mathrm{H}]=-0.20$ and therefore becomes more or less pronounced according to binning. We checked that this second peak is not related to the two bulge globular clusters NGC 6522 and NGC 6528 that are close to the region. The former has an intermediate metallicity close to -1 (Terndrup et al. 1998), while the latter, with a mean metallicity of -0.1 (Zoccali et al. 2004) is too far from the observed window.

To investigate the significance of low-level secondary peaks, we estimate the probability density function (PDF) from the raw data, using a kernel estimator. This estimator is

$\hat{\Phi}(x)=\frac{1}{n} \sum_{i=1}^{n} \frac{1}{h} K\left(\frac{x-X_{i}}{h}\right)$, 
Table 4. Statistics of the samples: the size $n$ (number of stars), the mean and median (in dex), the smoothing parameter $\hat{h}$ according to Sheather $\&$ Jones (1991), and the variability band width $\epsilon$.

\begin{tabular}{cccccc}
\hline \hline Subsample & $n$ & Mean & Median & $\hat{h}$ & $\epsilon$ \\
\hline$[\mathrm{Fe} / \mathrm{H}]$ & 219 & $0.05_{ \pm 0.03}$ & $0.16_{ \pm 0.04}$ & 0.0881 & 0.0605 \\
{$[\mathrm{Mg} / \mathrm{H}]$} & 162 & $0.14_{ \pm 0.02}$ & $0.21_{ \pm 0.03}$ & 0.0871 & 0.0707 \\
\hline
\end{tabular}

where $\left(X_{i}\right)$ are the observed metallicities, $n$ is the sample size, and $h$ the window width, also called the smoothing parameter. We chose the kernel function $K$ to be a Gaussian distribution. The smoothing parameter $h$ in Eq. (3) is estimated using the scheme described by Sheather \& Jones (1991). The estimator $\hat{h}$, as solution of Eq. (12) in Sheather \& Jones (1991), is given in Table 4 for each subsample.

The variability bands, as described by Bowman \& Azzalini (1997, Chap. 2.3), are computed to assess the significance of the modes in the derived distributions. In the case of a Gaussian kernel function, the variability band width around $\sqrt{\hat{\Phi}}$ is $\epsilon \approx$ $0.2656(\hat{h} n)^{-0.5}$ (Bowman \& Azzalini 1997). The values are given in Table 4 and are displayed in the lower panels in Fig. 10. More details about the smoothing method can be found in Royer et al. (2007, Sect. 4 and Appendix C).

As far as the secondary modes observed in the $[\mathrm{Fe} / \mathrm{H}]$ histogram (Fig. 10) are concerned, the smoothed distribution, together with the variability band, indicates that the most deficient one, around $[\mathrm{Fe} / \mathrm{H}]=-0.55 \mathrm{dex}$, is significant, whereas the intermediate one, around $[\mathrm{Fe} / \mathrm{H}]=-0.20 \mathrm{dex}$, is not and is instead part of a transition between the two main modes: around $[\mathrm{Fe} / \mathrm{H}]=0.35 \mathrm{dex}$ and $[\mathrm{Fe} / \mathrm{H}]=-0.55 \mathrm{dex}$.

\subsection{2. $[\mathrm{Mg} / \mathrm{H}]$ metallicity distribution}

With the analysis method described in Sect. 3.5, Mg abundances have been determined for a subsample of 162 red clump stars built by excluding stars with high uncertainties in the $[\mathrm{Fe} / \mathrm{H}]$ value (only stars with $\sigma_{\text {up }}(\mathrm{Fe})<0.30$ were kept). As illustrated in Fig. 10, the resulting $[\mathrm{Fe} / \mathrm{H}]$ distribution has the same global shape as the one found before from the total sample, as was confirmed by a Kolmogorov-Smirnov test $(D=0.0793, p$-value $=$ $0.5996)$. The main difference between the two distributions is for $[\mathrm{Fe} / \mathrm{H}]>0.2$ where our selection criteria has excluded a large number of stars, but the $[\mathrm{Fe} / \mathrm{H}]$ distribution for the 162 stars still shows the same sharp decrease in the number of stars at very high metallicities.

The resulting $[\mathrm{Mg} / \mathrm{H}]$ distribution is shown in Fig. 10 . Compared to the $[\mathrm{Fe} / \mathrm{H}]$ distribution, the $[\mathrm{Mg} / \mathrm{H}]$ distribution is much narrower, ranging from $[\mathrm{Mg} / \mathrm{H}]=-0.7$ to $[\mathrm{Mg} / \mathrm{H}]=+0.6$. While the sample contains $25 \%$ of stars with $[\mathrm{Fe} / \mathrm{H}]<-0.2$, those stars all have $[\mathrm{Mg} / \mathrm{Fe}]>0$, which explains the lack of the metal-poor tail of the $[\mathrm{Mg} / \mathrm{H}]$ distribution. The mean and median values are higher in $[\mathrm{Mg} / \mathrm{H}]$ than in $[\mathrm{Fe} / \mathrm{H}]: 0.14$ and 0.21 respectively (to be compared to 0.05 and 0.16 ). The distribution also shows two peaks: one around $[\mathrm{Mg} / \mathrm{H}]=+0.3$ and a second less significant around $[\mathrm{Mg} / \mathrm{H}]=-0.3$. For $[\mathrm{MgH}]>+0.3$, the number of stars decreases very abruptly. This characteristic has already been seen in the $[\mathrm{Fe} / \mathrm{H}]$ distribution but is even more drastic on the $[\mathrm{Mg} / \mathrm{H}]$ distribution. Moreover, we would expect this effect to be stronger with the addition of the stars excluded by the selection. Indeed, the excluded stars are mainly very high metallicity stars $([\mathrm{Fe} / \mathrm{H}]>0.2)$ that should follow the same $[\mathrm{Mg} / \mathrm{Fe}]$ trend as the other Fe-rich stars (see Fig. 14), which have $[\mathrm{Mg} / \mathrm{Fe}]$ abundances from -0.1 to 0.1 , so they should populate the region where $0.1<[\mathrm{Mg} / \mathrm{H}]<0.3$. This effect will be discussed further in Sect. 5 .

The smoothed distribution of $[\mathrm{Mg} / \mathrm{H}]$ does not display any significant secondary mode, but the spread of the distribution is indeed smaller than the one observed for $[\mathrm{Fe} / \mathrm{H}]$.

\subsection{3. $[\mathrm{Fe} / \mathrm{H}]$ distribution robustness}

To further investigate the robustness of our iron MD against abundance undertainties, we rejected stars with high uncertainties in two different ways, and checked the consistency of the resulting MD: $i)$ stars with $\sigma_{\mathrm{lo}}(\mathrm{Fe}) \leqslant 0.22$ were kept; $i$ i) stars with $\sigma_{\text {up }}(\mathrm{Fe}) \leqslant 0.28$ and $[\mathrm{Fe} / \mathrm{H}]<0.20$ or $\sigma_{\text {up }}(\mathrm{Fe}) \leqslant 0.36$ and $[\mathrm{Fe} / \mathrm{H}] \geq 0.20$ were kept, taking the increase in the $\sigma_{\mathrm{lo}}(\mathrm{Fe})$ uncertainty with metallicity into account (see Sect. 3.4 and Fig. 6). The two resulting distributions were found to be fully compatible with the total MD. Furthermore, to detect possible bias in the MD arising from the analysis method itself, we checked the robustness of the MD upon the difference between the spectroscopic and photometric $T_{\text {eff }}$. The resulting MD (obtained for the stars with a temperature difference $\leqslant 50 \mathrm{~K}$ and $\geqslant 150 \mathrm{~K}$ ) are also compatible with the total MD.

However, the decrease in the iron MD at the high-metallicity end (highlighted by the small number of stars with $[\mathrm{Fe} / \mathrm{H}]>0.5$ ) is too sharp to be compatible with the expected measurement errors (see 3.4 and Fig. 6) and suggests that a bias against the highest $[\mathrm{Fe} / \mathrm{H}]$ could be present. To show this, let us extract the stars from the MD with supersolar metallicities, as representative of the metal-rich peak of the distribution. First, using $[\mathrm{Fe} / \mathrm{H}]>0$ as a lower cut-off, the cumulative distribution of supersolar metallicities is plotted in Fig. 11. Under the hypothesis that, even if the highest metallicities are biased, the median of the distribution of the metal-rich peak is conserved (which is the case if errors remain almost symmetrical), this median (i.e., frequency $=0.5$ ), corresponds to $[\mathrm{Fe} / \mathrm{H}]=0.35 \mathrm{dex}$. This median is relatively robust to different cut-off values $(-0.1$ or $0.1 \mathrm{dex})$ for defining the high-metallicity peak, for which we obtain $[\mathrm{Fe} / \mathrm{H}]=0.32$ and 0.36 dex, respectively. The frequencies 0.16 and 0.84 mark the $1-\sigma$ positions from the median on the lower and upper sides of the distribution. These values, displayed in Fig. 11, show a clear asymmetry: $\sigma_{\text {left }}=0.21 \mathrm{dex}$ and $\sigma_{\text {right }}=0.16 \mathrm{dex}$. While $\sigma_{\text {left }}$ is slightly larger than the median expected measurement error $\sigma_{\text {low,Fe } / \mathrm{H}]>0}$ of 0.17 , allowing for a small astrophysical scatter, the $\sigma_{\text {right }}$ is too small compared to the median expected error $\sigma_{\text {up },[\mathrm{Fe} / \mathrm{H}]>0}=0.24$ (Fig. 6). The metal-rich end of the [Fe/H] MD is thus too steep to be consistent with the expected dispersion of the measurements due to their observational uncertainties alone. One possibility is that the remaining degeneracies between $\xi T_{\text {eff }}$ and $[\mathrm{Fe} / \mathrm{H}]$ introduce a bias in the metallicity estimates of the most metal-rich stars $([\mathrm{Fe} / \mathrm{H}]>0.35)$ at the resolution and $S / N$ of the present sample, preventing us to truly establish the underlying shape of the iron MD tail at high metallicities.

This bias is not detected in $[\mathrm{Mg} / \mathrm{H}]$, though, for which the observed $[\mathrm{Mg} / \mathrm{H}]$ distribution is compatible with the expected uncertainties, even if this distribution also displays a sharp cutoff at high metallicities. This is because the $\mathrm{Mg}$ lines used in the analysis are weak and do not suffer as much from $\xi T_{\text {eff }}$ degeneracies in the stellar parameter determination. As a result, the uncertainties on $[\mathrm{Mg} / \mathrm{H}]$ are both smaller than those on $[\mathrm{Fe} / \mathrm{H}]$ at high metallicities, and less asymmetric. Another way to say this is that, since the error on $[\mathrm{Mg} / \mathrm{H}]$ is dominated by actual line measurement errors (synthetic spectra fits in this case), the possible (small) bias in $[\mathrm{Mg} / \mathrm{H}]$ at the highest metallicities is hidden. 


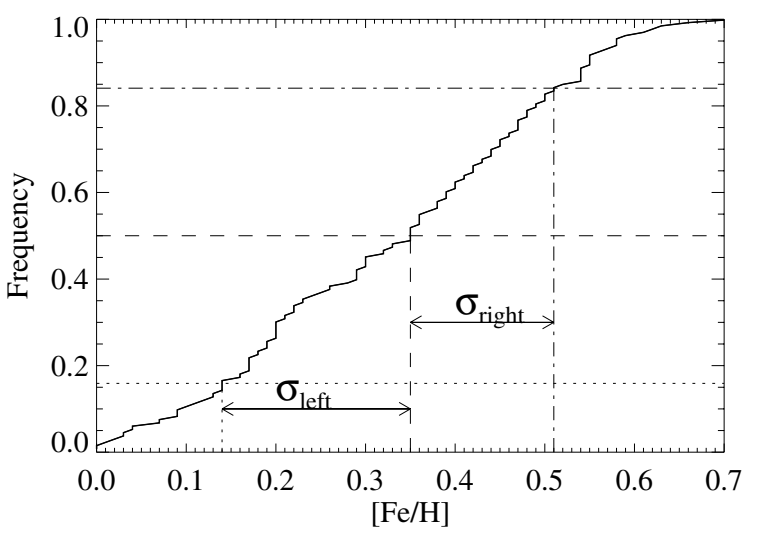

Fig. 11. The cumulative iron metallicity distribution for $[\mathrm{Fe} / \mathrm{H}]>0$ (solid line). The median and the left and right standard deviations are derived from the frequency values, assuming Gaussian errors.

To understand whether such a bias could indeed be present, we computed synthetic spectra of metal-rich $(0$. to +0.75 dex $)$ red clump stars, then sliced and convolved them to the same wavelength domains, resolution, and sampling as our observed GIRAFFE spectra. We added photon noise to the spectra to reproduce a set of spectra with $S / N=100,50,40$, and 30. (100 realisations of the noise were generated for each synthetic spectrum and noise level.) We then retrieved the stellar parameters and metallicity using the same method as for the bulge stars (equivalent width measurements and iteration on the stellar parameters). The results of these extensive simulations show that for supersolar metallicities, there is indeed a slight bias in the retrieved metallicities, although it is in the opposite direction to what would be suggested by the error analysis above: on average, very metal-rich stars are found to be slightly too metal-rich by our method. On average, a $[\mathrm{Fe} / \mathrm{H}]=+0.5$ star will be found +0.14 to +0.17 dex more metal-rich, whereas an $[\mathrm{Fe} / \mathrm{H}]=+0.25$ star will be found to be +0.10 to 0.14 dex more metal-rich (the bias steadily growing with decreasing $S / N$ from 100 to 30). At and below solar metallicities, the bias vanishes. Finally, we also confirmed this bias by performing the same test on the observed $\mu$ Leo spectrum, degraded in resolution, wavelength coverage, and photon noise. In this case, the bias is +0.13 at $S / N=50$ and +0.18 at $S / N=30$.

Based on these findings, we warn the reader that there may be a bias in the highest metallicity stars of the sample, although this bias is not understood well enough to be corrected.

\subsubsection{Comparison with the $[\mathrm{Fe} / \mathrm{H}]$ distribution of $\mathrm{RGB}$ stars}

In Zoccali et al. (2008), another bulge iron MD was obtained in Baade's window from a sample of 204 bulge red giants stars. This MD was found to agree with that of the the red clump stars, and the small differences between the two were found to be small enough to combine them to establish the Baade's window MD for the comparison with the other fields (see Sect. 6 of Zoccali et al. 2008). However, for other statistical works (see Babusiaux et al. 2010), the RGB and red clump MD had to be totally compatible to be combined, but a Kolmogorov-Smirnov (KS) test did not confirm that both samples could be drawn from a parent population $(p$-value $=0.0005)$. In contrast, the two samples were found to be slightly offset, with the RGB MD on average 0.07 more metal-poor than the red clump MD.

The difference between the two MD could arise from small differences in the analysis itself. Indeed, even if using the same

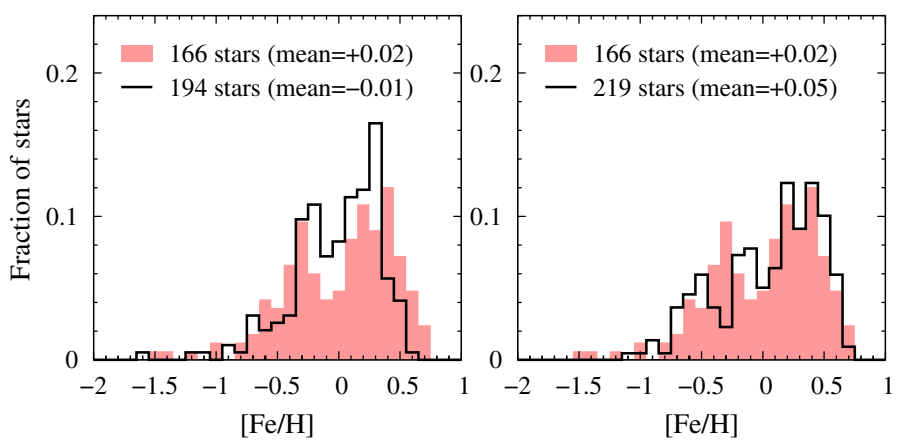

Fig. 12. Iron metallicity distribution (MD) of RGB stars obtained with the automatic procedure (red shaded histogram) compared with the MD of Zoccali et al. (2008) (left panel, black line) and with the MD of the red clump stars (right panel, black line). The new RGB MD is in total agreement with the red clump one (right panel) but is shifted toward high metallicities compared to the MD of Zoccali et al. (2008) (left panel). This small difference is due to the difference in analysis of the two samples as explained in the text.

criteria to determine the stellar parameters, the analysis of the RGB samples is different on two points: i) the photometric temperature is estimated from the $V-I$ index or from the TiO index (whereas it is computed from $V-I, V-J, V-K$ for the red clump sample), and ii) the stellar parameters are determined in a manual way (whereas the global procedure is automatic for the red clump sample). To investigate the two previous differences, we reanalysed the RGB sample again using the same automatic procedure with two values for the photometric temperature: i) the same as the one of Zoccali et al. (2008), and ii) a value computed from the indices $V-J, V-H, V-K$ (as for the red clump sample). From the 204 RGB stars sample, we excluded six stars as members of the globular cluster NGC 6522 and four as suspicious binaries. For the test ii), we also excluded stars for which the 2MASS survey only gives lower limits in one or more IR bands. Finally, $[\mathrm{Fe} / \mathrm{H}]$ values were obtained for 194 stars in case i) and 166 stars in case ii). Before comparing old and new $[\mathrm{Fe} / \mathrm{H}]$ values, we checked that the MD of Zoccali et al. (2008) reduced to the 166 stars of ii) was fully compatible with the total one (194 stars). As shown by Fig. 12, the MD obtained for the 166 stars from the automatic procedure has a global shape close to the one of Zoccali et al. (2008, see Fig. 12) with many more stars at very high metallicities $([\mathrm{Fe} / \mathrm{H}]>0.4)$ leading to mean and median values ( 0.02 and 0.11 respectively) slightly higher than values previously found ( -0.01 and 0.05 , respectively). However, in cases i) et ii), from the results of a KS test, the MD are not compatible with the one of Zoccali et al. (2008): $p$-value $=0.03$ for i) and $p$-value $=0.05$ for ii), but they are compatible with the red clump one: $p$-value $=0.65$ for i) and $p$-value $=0.58$ for ii) as illustrated by Fig. 12 in case ii). These results show that the difference between the MD of Zoccali et al. (2008) and the red clump sample comes from the automatic procedure itself rather than from the initial photometric temperature adopted. However, the difference between the RGB MD presented here and that of Zoccali et al. (2008) remains very small, and that the conclusions of their paper remain fully valid.

\subsection{Unravelling two populations}

\subsubsection{The deconvolved metallicity distribution}

From the PDFs estimated in Sect. 4.1.1 and plotted in Fig. 10, one can rectify the error law effect. However, we argued in 


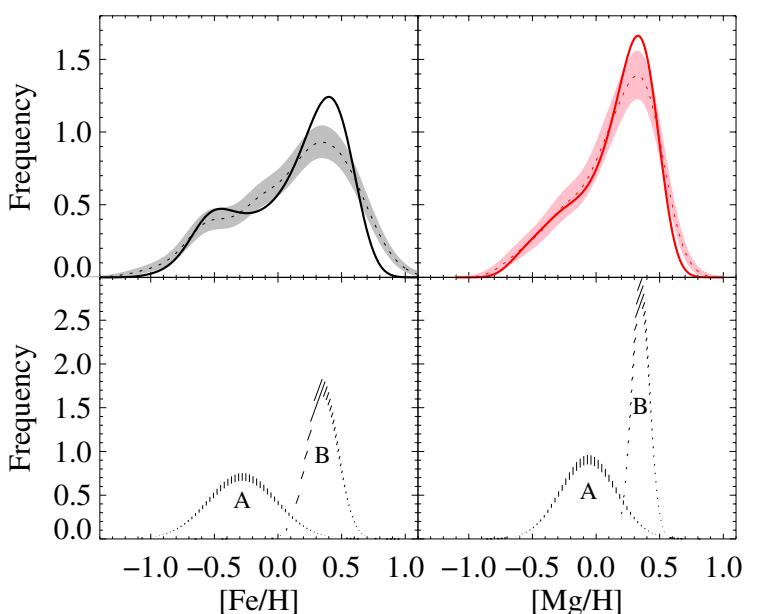

Fig. 13. Upper left panel: smoothed distribution of $[\mathrm{Fe} / \mathrm{H}]$, as in Fig. 10, with the resulting deconvolved distribution using a constant error (solid line). Upper right panel: smoothed distribution of $[\mathrm{Mg} / \mathrm{H}]$, as in Fig. 10 with the resulting deconvolved distribution using a constant error (solid line). Lower left panel: resulting Gaussian populations in the $[\mathrm{Fe} / \mathrm{H}]$ distribution. See Table 5 for the parameters of the Gaussians. The error bars represent the uncertainty in the amplitude of the Gaussian populations. Lower right panel: resulting Gaussian populations in the $[\mathrm{Mg} / \mathrm{H}]$ distribution.

Sect. 4.1.3 that the metal-rich end of the iron metallicity distribution may be biased at the highest metallicities. To avoid any spurious result in deconvolving the observed PDF, we chose to stretch the metal-rich side of the MDF, so that $\sigma_{\text {right }}$ finally matches $\sigma_{\text {left }}$. The stretched distribution is superimposed in Fig. 10. This stretching will not erase any bias in the highest metallicities if it is indeed present, but helps the deconvolution algorithm to perform nominally.

We used the Lucy-Richardson deconvolution technique (Lucy 1974; Richardson 1972) to recover the rectified MD. More details about the deconvolution method can be found in Royer et al. (2007, Sect. 4 and Appendix C).

In this work, the error law is assumed to be Gaussian. We chose to model the error law as a constant, using the median of the $\left(\sigma_{\text {low }}(\mathrm{Fe})+\sigma_{\text {up }}(\mathrm{Fe})\right) / 2$ and $\sigma_{\text {low }}(\mathrm{Mg}): 0.21$ dex and 0.14 dex, respectively. These values are used to estimate the standard deviation in the Gaussian error distribution during the LucyRichardson deconvolution. The stopping criterion described by Lucy (1994) was used, and the resulting number of iterations were 11 and 5 for the iron and magnesium distributions, respectively. The resulting PDF are plotted in Fig. 13. The deconvolved $[\mathrm{Fe} / \mathrm{H}]$ distribution shows a clear bimodality with a lowmetallicity peak around -0.45 dex and a high-metallicity peak around +0.3 dex. The deconvolution does not show any clear features in the resulting distribution for $[\mathrm{Mg} / \mathrm{H}]$. This is because $[\mathrm{Mg} / \mathrm{H}]$ abundances are less spread out and their associated errors smaller.

\subsubsection{Gaussian mixture}

The metallicity distributions described in Sect. 4.1 suggest that they may be made up of contributions from different stellar populations. In a first attempt to identify possible substructures, we have decomposed the observed $[\mathrm{Fe} / \mathrm{H}]$ and $[\mathrm{Mg} / \mathrm{H}]$ distributions into a finite number of Gaussian components. The SEMMUL algorithm, developed by Celeux \& Diebolt (1986), was applied. SEMMUL (Stochastic, Estimation, Maximisation,
Table 5. Separation of the sample in two Gaussian components (A and B), with $[\mathrm{Fe} / \mathrm{H}],[\mathrm{Mg} / \mathrm{H}]$ or both simultaneously as discriminant variables.

\begin{tabular}{|c|c|c|c|c|c|}
\hline & $\overline{[\mathrm{Fe} / \mathrm{H}]}$ & $\sigma_{[\mathrm{Fe} / \mathrm{H}]}$ & $\overline{[\mathrm{Mg} / \mathrm{H}]}$ & $\sigma_{[\mathrm{Mg}}$ & $\%$ \\
\hline \multicolumn{4}{|c|}{ 1D-separation along $[\mathrm{Fe} / \mathrm{H}]$} & \multicolumn{2}{|c|}{$N_{\text {stars }}=219$} \\
\hline A & $-0.30_{ \pm 0.03}$ & $0.25 \pm 0.01$ & & & $45 \pm 3$ \\
\hline B & $0.32 \pm 0.01$ & $0.11_{ \pm 0.01}$ & & & $55_{ \pm 3}$ \\
\hline \multicolumn{4}{|c|}{ 1D-separation along $[\mathrm{Mg} / \mathrm{H}]$} & \multicolumn{2}{|c|}{$N_{\text {stars }}=162$} \\
\hline A & & & $-0.06 \pm 0.02$ & $0.22 \pm 0.01$ & $49_{ \pm 4}$ \\
\hline B & & & 0.35 & $0.07_{ \pm 0.01}$ & $51_{ \pm 4}$ \\
\hline \multicolumn{4}{|c|}{ 2D-separation in $([\mathrm{Fe} / \mathrm{H}],[\mathrm{Mg} / \mathrm{H}])$} & \multicolumn{2}{|c|}{$N_{\text {stars }}=162$} \\
\hline A & $-0.27 \pm 0.03$ & $0.31_{ \pm 0.02}$ & $-0.04_{ \pm 0.03}$ & $0.25_{ \pm 0.01}$ & $53 \pm 4$ \\
\hline B & $0.29 \pm 0.01$ & $0.12 \pm 0.01$ & $0.36 \pm 0.01$ & $0.07 \pm 0.01$ & $47 \pm 4$ \\
\hline
\end{tabular}

Notes. $\sigma_{[\mathrm{Fe} / \mathrm{H}]}$ and $\sigma_{[\mathrm{Mg} / \mathrm{H}]}$ are the cosmic dispersion of the population.

MULtidimensional) is an iterative method for numerically approximating maximum likelihood estimates for incomplete data with a stochastic step to accelerate the convergence. It allows separation of Gaussian components without prior knowledge of the number of components. Only a maximum number is necessary. In addition, it does require defining a set of initial conditions. The algorithm has been modified by Arenou (1993) to take observational errors into account, thus the modified version allows estimating the cosmic dispersion of the population.

SEMMUL has been applied to the 1 -dimensional $[\mathrm{Fe} / \mathrm{H}]$ and $[\mathrm{Mg} / \mathrm{H}]$ distributions separately, and to the 2-dimensional joint distribution $([\mathrm{Fe} / \mathrm{H}],[\mathrm{Mg} / \mathrm{H}])$. Thousands of runs were carried out in each case, assuming that the sample is a mixture of 2 or 3 discrete components. In all cases, the algorithm converges to a two-component solution with stable results, dividing the sample in roughly two equal-sized populations.

The resulting two components (that we call $\mathrm{A}$ and $\mathrm{B}$ ) are summarised in Table 5. For each component, the mean chemical composition, the dispersion, and the fraction (in percent) of stars in each group are given with the corresponding standard errors. The $\mathrm{A}$ and $\mathrm{B}$ population characteristics are very consistent among the three separations (1-dimensional along $[\mathrm{Fe} / \mathrm{H}]$ or $[\mathrm{Mg} / \mathrm{H}]$, or 2-dimensional).

In Sect. 4.1.3 we argued that the high-metallicity tail of the iron distribution may be biased. In this context, we may wonder whether the mean values and dispersions obtained in the Gaussian decompositions of population B are realistic. The comparison between the estimated mean values $([\mathrm{Fe} / \mathrm{H}]=0.32$ and 0.29 , see Table 5), and the median values obtained from the cumulative metallicity distribution (for $[\mathrm{Fe} / \mathrm{H}]>0$ ) in Sect. $4.1 .3([\mathrm{Fe} / \mathrm{H}]=0.32$ to 0.36$)$ show that they are consistent. On the other hand, the obtained cosmic dispersions of population B (0.11-0.12 dex) could arguably be underestimated. However, according to the same cumulative distribution presented in Sect. 4.1.3, the total metallicity dispersion is about 0.2 dex in this population, and the expected measurement errors are similar, which implies that the cosmic dispersion of the population is small, as obtained by our algorithm.

\subsubsection{Characterising the two populations}

Two populations are clearly identified by the Gaussian separation exercise. The metal-poor component $(\mathrm{A})$ is centred on $[\mathrm{Fe} / \mathrm{H}]=-0.30$ and $[\mathrm{Mg} / \mathrm{H}]=-0.06$, with a large dispersion ( $\sim 0.3$ dex $)$, while the metal-rich component (B) has similar mean $\mathrm{Fe}$ and $\mathrm{Mg}$ content $([\mathrm{Fe} / \mathrm{H}]=0.32$ and $[\mathrm{Mg} / \mathrm{H}]=+0.35)$ and $\mathrm{a}$ very small dispersion $(0.11$ in $[\mathrm{Fe} / \mathrm{H}]$ and 0.07 in $[\mathrm{Mg} / \mathrm{H}])$. 


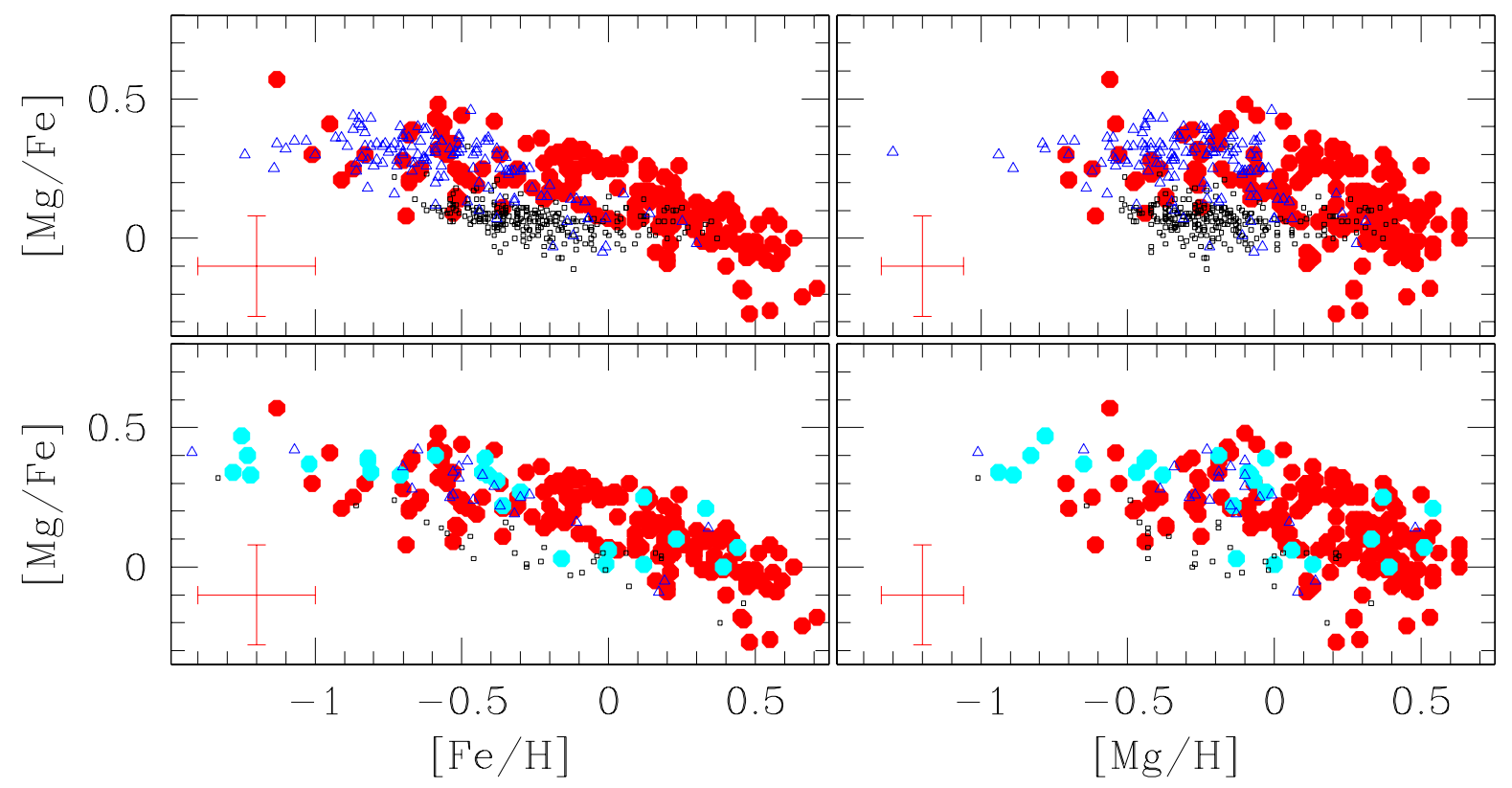

Fig. 14. $[\mathrm{Mg} / \mathrm{Fe}]$ as a function of $[\mathrm{Fe} / \mathrm{H}]$ (right panel) and $[\mathrm{Mg} / \mathrm{H}]$ (left panel) for our bulge red clump sample (red points) compared with thick (blue) and thin (black) discs stars. In the two upper panels, the disc reference samples are from the main sequence stars studies of Bensby et al. (2005) (triangles), Reddy et al. (2006) (squares), Fuhrmann (2008) (crosses), whereas the two lower panels show the solar neighbourhood disc giants by Alves-Brito et al. (2010), as well as their bulge giants (large filled cyan circles). In the low metallicity range $([\mathrm{Fe} / \mathrm{H}]<-0.3$ and $[\mathrm{Mg} / \mathrm{H}]<0)$, the thick disc and the bulge show similar $[\mathrm{Mg} / \mathrm{Fe}]$ ratios, higher than in thin disc stars.

The dispersion of population B is very small, comparable in fact to the metallicity dispersion of the Galactic disc in the solar neighbourhood (or even smaller). The more metal-poor population $\mathrm{A}$ is, on the contrary, quite extended, both in $[\mathrm{Fe} / \mathrm{H}]$ and in $[\mathrm{Mg} / \mathrm{H}]$. This dispersion could in part be due to contamination (see Sect. 2.2) by the thick disc in the inner Galactic regions, which could account for $\sim 10-15 \%$ of population A $(5.9 \%$ of the total sample). In fact, we see in the next section that the chemical signature of this population (A) is not distinct from that of the thick disc, and we can therefore not exclude that part of it is made up of thick disc stars, indistinguishable from the bona-fide bulge population by any of our observables.

It is further interesting to note that the two components thus separated are more widely separated in $[\mathrm{Fe} / \mathrm{H}]$ than in $[\mathrm{Mg} / \mathrm{H}]$, as reflected by the different mean corresponding $[\mathrm{Mg} / \mathrm{Fe}]$ ratios (see Sect. 4.3 and Fig. 14), hinting at two physically separated populations. While the metal-poor component $\mathrm{A}$ has a clearly defined magnesium overabundance of $[\mathrm{Mg} / \mathrm{Fe}] \sim 0.24$, the metal-rich component $\mathrm{B}$ has a significantly lower $[\mathrm{Mg} / \mathrm{Fe}] \sim$ 0.05 , not significantly different than the solar neighbourhood $\operatorname{disc}[\mathrm{Mg} / \mathrm{Fe}]$.

We tentatively explain the existence of these bulge populations as the result of different origins. In Babusiaux et al. (2010), we combine the abundances and the kinematics (radial velocities plus proper motions from OGLE-II) and show a significant difference in the kinematics of the metal-rich and the metal-poor components. The velocity ellipsoid of the metal-rich component shows a vertex deviation consistent with what is expected from a population kinematically supporting a bar. The metal-poor component, on the other hand, shows no vertex deviation, consistent with an old spheroidal population. We therefore relate the richest population to a bar-driven pseudo-bulge and the more metal-poor one to an old spheroid with a rapid time-scale formation. Similar results have been found by Soto et al. (2007) and Zhao et al. (1994), based on kinematics and low-resolution metallicities.
We note finally that this mixture of populations in Baade's window is fully compatible with the variation in the metallicity distribution as a function of Galactic latitude noticed by Zoccali et al. (2008) (from $b=-4$ to $-12^{\circ}$ ), which we would therefore attribute to the gradual disappearance of the pseudo-bulge component $\mathrm{B}$ as one moves away from the Galactic plane, while the old spheroid (A) would dominate out to higher latitudes. The absence of gradients in the inner bulge found by Rich et al. (2007) is also consistent, as this region would be dominated only by component $\mathrm{B}$. We also note that population $\mathrm{B}$ has to be predominantly old (perhaps not surprisingly, if disc formation occurs inside out), given that Clarkson et al. (2008) find a pure old population in a field at $b=-2.65^{\circ}$ (i.e., significantly closer to the plane than Baade's window).

\subsection{The $[\mathrm{Mg} / \mathrm{Fe}]$ trend in the bulge}

Our $\mathrm{Mg}$ results in terms of $[\mathrm{Mg} / \mathrm{Fe}]$ against $[\mathrm{Fe} / \mathrm{H}]$ and $[\mathrm{Mg} / \mathrm{H}]$ are shown in Fig. 14. The $[\mathrm{Mg} / \mathrm{Fe}]$ ratios in the bulge decreases with both $[\mathrm{Fe} / \mathrm{H}]$ or $[\mathrm{Mg} / \mathrm{H}]$ from $\sim+0.5$ to near solar values. Comparing our results with the recent optical and IR studies of bulge giants from Rich \& Origlia (2005), Fulbright et al. (2007), and Rich et al. (2007) on the common metallicity ranges, we note that the general trend is similar, although our much larger statistics allow us to define the trend with more confidence. Our derived $[\mathrm{Mg} / \mathrm{Fe}]$ trend is also in perfect agreement with the recent work of Gonzalez et al. (2011) based on $~ 650$ RGB stars at four different locations in the bulge analysed in a very similar fashion to our RC sample. Gonzalez et al. (2011) measured not only $\mathrm{Mg}$ but also three other $\alpha$-elements $(\mathrm{Ca}, \mathrm{Si}, \mathrm{Ti})$, showing the robustness of the derived $[\mathrm{Mg} / \mathrm{Fe}]$.

To understand the relation that the bulge bears to the Galactic $\operatorname{disc}(\mathrm{s})$, it is interesting to compare the $[\mathrm{Mg} / \mathrm{Fe}]$ ratios in the bulge sample to the abundance trends found in the Galactic thin and thick discs. In Fig. 14, we plotted the thin and thick 
disc stars abundances from the studies of Bensby et al. (2005); Reddy et al. (2006) and Fuhrmann (2008). These studies are based on very careful and thourough analysis of large samples of dwarf stars in the solar neighbourhood. As recently pointed out by Meléndez et al. (2008) and Alves-Brito et al. (2010), it would be better, for the sake of minimising possible systematics in the abundance analysis, to compare bulge giants with solarneighbourhood thin and thick disc giants observed and analysed in the same conditions. However, from a statistical point of view, the available samples of local giants available for such comparisons is still quite small (Alves-Brito et al. 2010, 20/30 thick/thin disc stars, respectively). We therefore decided to stick to a comparison with local disc dwarfs, once a number of verifications had been performed: $(i)$ we analysed the $[\mathrm{Mg} / \mathrm{Fe}]$ of two reference stars, $\mu$ Leo and Arcturus $([\mathrm{Mg} / \mathrm{Fe}]=+0.12$ and +0.38 ), which respectively fall on top of their parent population as derived from local dwarf samples; (ii) Fulbright et al. (2007) analysed a sample of local $\mathrm{K}$ giants, and found no difference between the $[\mathrm{Mg} / \mathrm{Fe}]$ trend derived from these giants and those defined from local dwarfs; (iii) Mishenina et al. (2006) also analysed a large sample of local red clump giants, and reach a similar conclusion for $[\mathrm{Mg} / \mathrm{Fe}]$. From these checks, we conclude that it is fair to compare the $[\mathrm{Mg} / \mathrm{Fe}]$ abundances for the bulge red clump giants presented here to those of local disc dwarfs. In Fig. 14, the two lower panels show that indeed our conclusions would not be altered in any way if comparing our sample to local giants (Alves-Brito et al. 2010). We do, however, caution that, in the highest metallicity regime, systematic differences from 0.10 to 0.15 on the $[\mathrm{Mg} / \mathrm{Fe}]$ trends are found between different studies of dwarfs stars. This is clearly a domain where abundances have to be taken with caution in general, and even more so when they are derived, as here, from moderate $S / N$ and resolution spectra.

When compared to the local Galactic disc abundances, these various bulge samples show some differences depending on the metallicity range:

In the range $[\mathrm{Fe} / \mathrm{H}] \lesssim-0.3$, the number of stars in the present sample has increased dramatically over our previous UVES sample (Fig. 7) and they clearly show $[\mathrm{Mg} / \mathrm{Fe}]$ similar to those of the thick disc stars for all $[\mathrm{Fe} / \mathrm{H}]$. In the same metallicity range, our results differ from those of Fulbright et al. (2007) who find higher $[\mathrm{Mg} / \mathrm{Fe}]$ values than ours and a $[\mathrm{Mg} / \mathrm{Fe}]$ trend higher than that of their sample of disc giants. In fact, if we use Arcturus as a reference to insure that the results of Fulbright et al. (2007) are on the same scale as the present work, an offset of $\sim 0.10$ is expected $\left([\mathrm{Mg} / \mathrm{Fe}]_{\text {Fulbright }}-[\mathrm{Mg} / \mathrm{Fe}]_{\text {present }}=+0.11\right)$, bringing the $[\mathrm{Mg} / \mathrm{Fe}]$ of the two studies into agreement.

In the range $-0.3 \lesssim[\mathrm{Fe} / \mathrm{H}] \lesssim+0.1$, the bulge $[\mathrm{Mg} / \mathrm{Fe}]$ trend from our sample is clearly distinct and higher than that of the thin disc, regardless of the comparison sample used. In the same metallicity range, our bulge stars show $[\mathrm{Mg} / \mathrm{Fe}]$ on average higher than those of the thick disc stars. This confirms, with better statistics our previous results from the UVES sample (Lecureur et al. 2007), as well as the results of Fulbright et al. (2007). Given the residual systematic effect on the $[\mathrm{Mg} / \mathrm{Fe}]$ determination from GIRAFFE and UVES spectra for the stars in common (see Fig. 8), the difference between thick disc and bulge would be even more pronounced if we adopted the UVES abundances as the reference. However, this difference between bulge and thick disc has to be viewed with caution because of how few thick disc stars there are in this metallicity range and of the still debated thick-disc nature of this high-metallicity tail. Indeed, Bensby et al. $(2005,2007)$ argue that the thick disc extends at least to solar metallicities and shows a clear decrease in $[\alpha / \mathrm{Fe}]$ with increasing iron content for stars with $[\mathrm{Fe} / \mathrm{H}] \gtrsim-0.3$ denoting an extended star formation period (SNIa enrichment). In contrast, several authors (Reddy et al. 2006; Fuhrmann 2008) argue that the thick disc does not extend significantly in this metallicity regime and that there is no evidence of decreasing $[\alpha / \mathrm{Fe}]$ in the thick disc at all.

In the range $[\mathrm{Fe} / \mathrm{H}] \gtrsim+0.1$, the bulge shows $[\mathrm{Mg} / \mathrm{Fe}]$ ratios that are similar to those of the local thin disc, solar on average, and with a decreasing $[\mathrm{Mg} / \mathrm{Fe}]$ trend with increasing metallicity. This result confirms, but with better statistics, the results found for the oxygen by Meléndez et al. (2008) in the same metallicity regime. In contrast, our bulge $[\mathrm{Mg} / \mathrm{Fe}]$ ratios are significantly lower than those of Fulbright et al. (2007), even if allowing a shift of the latter by $\sim-0.10$ to insure that both studies lie on the same scale. We further note that Fulbright et al. (2007) derive $[\mathrm{Mg} / \mathrm{Fe}]=+0.02$ for $\mu \mathrm{Leo}$, 0.20 lower than what we found for this star (Lecureur et al. 2007). At such high metallicities, both $\mathrm{Fe}$ and $\mathrm{Mg}$ measurements remain quite uncertain, which could explain part of the difference between our study and the one of Fulbright et al. (2007). Moreover, the comparison of the bulge and disc trends should be taken with caution in that metallicity regime, since, as pointed out above, systematic differences between 0.10 and 0.15 are found between the $[\mathrm{Mg} / \mathrm{Fe}]$ trends at supersolar metallicities between different studies. More specifically, taking the local disc sample of giants by Fulbright et al. (2007) as a comparison, we would find that the $[\mathrm{Mg} / \mathrm{Fe}]$ of the bulge lies above that of the local disc, at variance with the conclusion that is drawn from the comparison to local dwarfs. However, we do not favour this interpretation since Fulbright et al. (2007) also finds $\mu$ Leo +0.11 dex lower in $[\mathrm{Mg} / \mathrm{Fe}]$ than we do.

Very recently, several papers have measured detailed abundances of a few bulge dwarfs from high-resolution and high $S / N$ spectra obtained during a gravitational microlensing event (Bensby et al. 2010b, 2009b,a; Cohen et al. 2008, 2009; Johnson et al. 2007, 2008). There are in total by now 14 microlensed dwarfs and subgiants analysed in the Galactic bulge (Bensby et al. 2010b), and although the early (2007 and 2008) microlens events yielded a sample of stars almost exclusively metal-rich that was irreconcilable with the MDF of bulge giants (Cohen et al. 2008, 2009; Epstein et al. 2010), the 2009 and 2010 events have uncovered a significant metal-poor population, taming down this conclusion to the point that Bensby et al. (2010b) claims that the two distributions could statistically arise from the same parent population, albeit a rather low probability. The current MDF from these microlensed unevolved stars is now highly bimodal, with a peak at $[\mathrm{Fe} / \mathrm{H}] \sim+0.3$ and another one at $[\mathrm{Fe} / \mathrm{H}] \sim-0.5 \mathrm{dex}$. This is very similar to the peaks uncovered by our population separation in the red clump sample. Interestingly, the metal-rich (similar to our population A) and metal-poor (similar to our population B) microlensed stars also have different ages, the latter being a clearly old population, while the former spans a range of ages (2-13 Gyr). This is again compatible with our suggestion that population $\mathrm{B}$ is related to the old bulge, while population $\mathrm{A}$ is kinematically related to a bar and would be made of a pre-existing disc. Even more interestingly perhaps, we are now in a position to compare the $[\mathrm{Mg} / \mathrm{Fe}]$ trends in the bulge from dwarf and giants stars. As for our bulge giants, $[\mathrm{Mg} / \mathrm{Fe}]$ in dwarfs overlays the local thin disc at super-solar metallicities, 

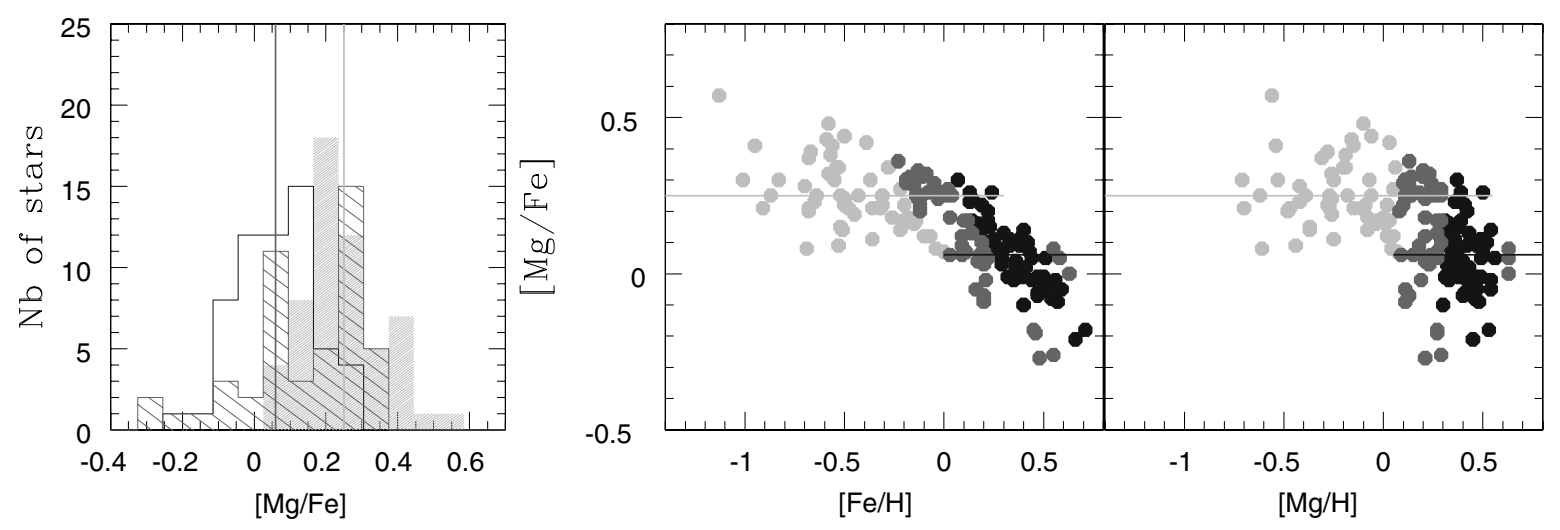

Fig. 15. Left panel: $[\mathrm{Mg} / \mathrm{Fe}]$ distribution for the stars with very high probabilities (>85\%) to belong to population A (empty histogram) and B (shaded histogram). The two lines show the peaks of these two distributions: 0.05 and 0.25 respectively. The hatched histogram represents the stars with low probabilities to belong to one or the other population. Right panels: $[\mathrm{Mg} / \mathrm{Fe}]$ as a function of $[\mathrm{Fe} / \mathrm{H}]$ and $[\mathrm{Mg} / \mathrm{Fe}]$ for the three groups of stars.

and the local thick disc at metallicities $[\mathrm{Fe} / \mathrm{H}] \leq-0.3$. The current sample of microlensed giants only contains two stars in the intermediate metallicity range (Bensby et al. 2010b), where we find the bulge to be richer in $[\mathrm{Mg} / \mathrm{Fe}]$ than both the local thin and thick discs. These two stars seem to trace the upper envelope of the local thick disc. To draw any further conclusion on this metallicity range, more microlensed dwarfs will need to be analysed.

To follow up on our tentative sample separation in two distinct populations we colour-coded in Fig. 15, the stars belonging to the populations $\mathrm{A}$ and $\mathrm{B}$ as separated in Table 5 along $[\mathrm{Mg} / \mathrm{H}]$, using the probability with which a star was assigned to its population. Stars with a very high probability $(>85 \%)$ to belong to population A are coded in light grey, while those with a high probability to belong to B are coded in black. Stars with a low probability of belonging to either populations are coded in dark grey. Population A shows high $[\mathrm{Mg} / \mathrm{Fe}]$ ratios, with a mean of $[\mathrm{Mg} / \mathrm{Fe}]=0.25$, while population $\mathrm{B}$ has lower $\mathrm{Mg}$ enhancements, close to solar, as discussed above. The intermediate $\mathrm{RC}$ population (those stars that could not be unambiguously classified) has both high and low $[\mathrm{Mg} / \mathrm{Fe}]$. In fact, the $[\mathrm{Mg} / \mathrm{Fe}]$ histogram of this population shows two well-defined peaks, one at +0.25 and the other at +0.05 , coinciding precisely with the means for populations A and B, respectively. It therefore seems that this intermediate population is a mix of stars with the same chemical properties of A or B population, rather than a smooth transition between the two. This is also visible in the run of $[\mathrm{Mg} / \mathrm{Fe}]$ with metallicity (whether $[\mathrm{Fe} / \mathrm{H}]$ or $[\mathrm{Mg} / \mathrm{H}]$, Fig. 15) at intermediate metallicities, where the stars seem to cluster around two discrete $[\mathrm{Mg} / \mathrm{Fe}]$ values extending the trends of populations $\mathrm{A}$ and $\mathrm{B}$, rather than following a smooth decreasing trend. As a result, we argue that population $\mathrm{A}$, which we associate with the old $\mathrm{Mg}$-enhanced bulge, extends up to metallicities of at least $[\mathrm{Fe} / \mathrm{H}]=+0.1$ and $[\mathrm{Mg} / \mathrm{H}]=+0.35$, and possibly up to $[\mathrm{Fe} / \mathrm{H}]=+0.25$ and $[\mathrm{Mg} / \mathrm{H}]=+0.5$. Conversely, population $\mathrm{B}$ probably extends down to $[\mathrm{Fe} / \mathrm{H}] \sim[\mathrm{Mg} / \mathrm{H}]=0.0$, with modest $\mathrm{Mg}$ enhancements. This will have an impact on the chemical evolution models that may be appropriate for representing these two populations.

\section{Chemical evolution and bulge formation}

In this section, with the aim of understanding the formation history and chemical evolution of the Galactic bulge, we compared our bulge MD with the results of two recent bulge formation models: the chemical evolution model of Ballero et al. (2007b) and the chemodynamical model of Immeli et al. (2004).

\subsection{Comparison to a chemical evolution model of the bulge}

The upper panel of Fig. 16 shows the MD predicted by the model of Ballero et al. (2007b), together with our observed iron MD. For the sake of comparison, the model MD has been convolved with the mean $[\mathrm{Fe} / \mathrm{H}]$ uncertainties of the observed sample (Fig. 6). By constraining their model parameters from bulgeobserved abundance ratios and Fe MD, Ballero et al. (2007b) conclude that the bulge formed in a very short time, with a high star formation efficiency ( $\mu \simeq 20 \mathrm{Gyr}^{-1}$ ) and an IMF flatter than in the solar neighbourhood. The MD they predict peaks around solar metallicity and is shifted toward lower metallicities with a more extended metal-poor tail with respect to our bulge observed MD. This disagreement was expected given the differences between our bulge MD and the one of Zoccali et al. (2003) and Fulbright et al. (2006), which were used as references by Ballero et al. (2007b). In fact, to predict near solar metallicities MD consistent with high $\alpha$-elements ratios, Ballero et al. (2007b) had to assume a flatter IMF than in the solar neighbourhood but also (see Ballero et al. 2007a) flatter than the stellar IMF of Kroupa (2001) and the IMF integrated over galaxies of Weidner \& Kroupa (2005). According to the tests done by the authors, to shift the predicted MD to more metal-rich (as observed in the present paper), the IMF should be further flattened. However, for an IMF slope lower than 0.35 , the position of the peak of the predicted MD becomes insensitive to further slope decreases. Moreover, a flatter IMF would in turn lead to predict higher $[\mathrm{Mg} / \mathrm{Fe}]$ and $[\mathrm{O} / \mathrm{Fe}]$ ratios than observed. Thus, it seems there is no longer any appropriate set of fundamental parameters for the bulge chemical model of Ballero et al. (2007b) to reproduce both our red clump MD and the $[\mathrm{Mg} / \mathrm{Fe}]$ (or $[\mathrm{O} / \mathrm{Fe}]$ ) trend observed in the bulge.

On the other hand, if we consider the observed MD as the result of two populations (see Sect. 4.2), it would be more appropriate to compare the MD of Ballero et al. (2007b) (given their bulge chemical model formation hypothesis) with the one associated to the old bulge component. This comparison is illustrated by Fig. 16 where the old bulge population (A) is modelled by a Gaussian with a mean $[\mathrm{Fe} / \mathrm{H}]$ of -0.3 and a width of 0.25 . With 

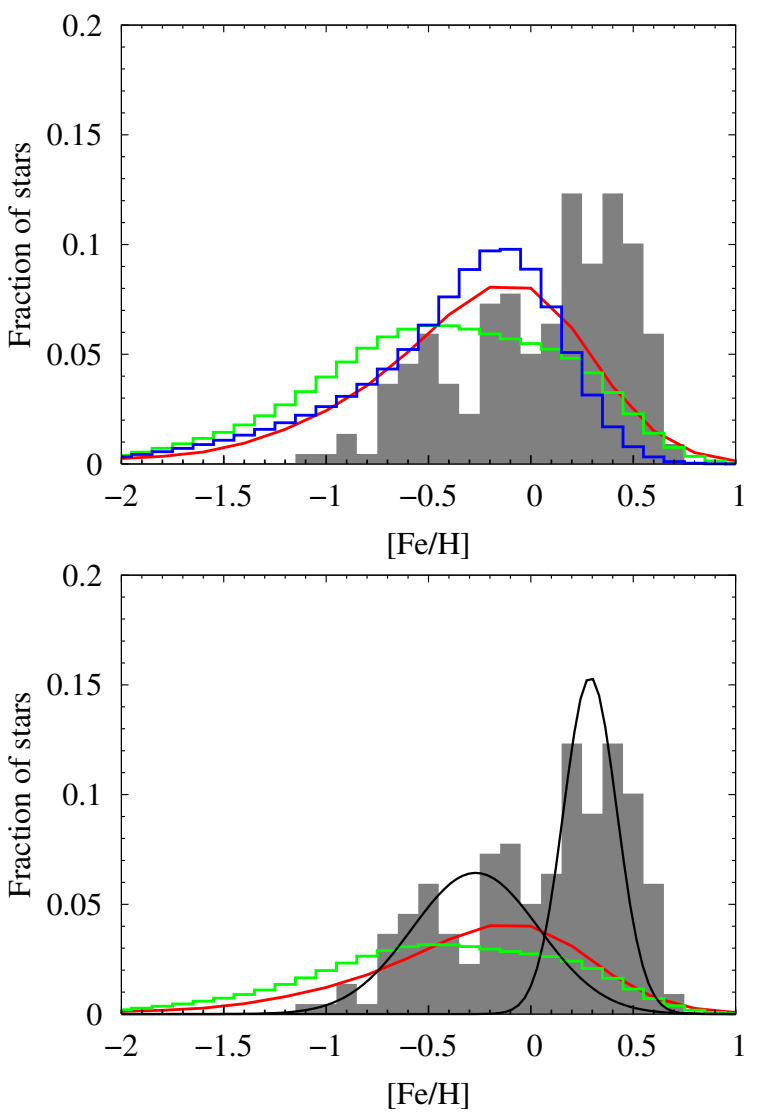

Fig. 16. Upper panel: comparison between the bulge MD predicted by the chemical model of Ballero et al. (2007b) (red line) and by the model of Immeli et al. (2004) (green line for the model B and blue line for the model F ) with the observed MD (shaded histogram). The three model MDs have been convolved with observational errors. Lower panel: the previous model MDs were normalised to $50 \%$ and compared to the MD of the two subpopulations A and B (each of them corresponding to 50\% of the total) illustrated here by two Gaussians with position and dispersion according to Table 5 .

its actual parametrisation, the model of Ballero et al. (2007b) also fails to reproduce the metal-poor population of the bulge. The predicted MD peaks at $[\mathrm{Fe} / \mathrm{H}]$ values higher than the mean of population $\mathrm{A}$, and is wider than the Gaussian. It underpredicts the fraction of stars in population $\mathrm{A}$ at intermediate metallicities $(-0.8<[\mathrm{Fe} / \mathrm{H}]<-0.1)$ and predicts a longer low-metallicity tail $([\mathrm{Fe} / \mathrm{H}]<-0.8)$. However, based on the parameter variations presented by Ballero et al. (2007b), we would expect that it is possible to find a set of parameters that would reproduce both the high $[\mathrm{Mg} / \mathrm{Fe}]$ and metallicity distribution of population $\mathrm{A}$. In particular, there would no longer be any need for a flatter IMF in the bulge.

\subsection{Comparison to a chemodynamical model}

The lower panel of Fig. 16 shows the comparison between our bulge iron MD and the predicted MD from two models ( $\mathrm{B}$ and F) of Immeli et al. (2004) convolved with the observational errors. Immeli et al. (2004) investigated different scenarios of bulge, disc and halo population formation from the evolution of a starforming disc with a chemodynamical 3D model that includes initial conditions from $\Lambda \mathrm{CDM}$ results and a two-phase (gas and stars) interstellar medium. The Galactic bulge is formed in very different ways depending on values adopted for the efficiency of energy dissipation of the cold cloud component from which stars form. With a high efficiency of energy dissipation, a massive bulge is formed from the central merging of clumps of stars and gas at relatively early times (model B). In contrast, with low efficiency values, the instability occurs in the stellar disc at a later time, forming a stellar bar that evolves and forms a pseudo-bulge (model F).

The two predicted MD are completely different from the observed one. As already explained in the previous section, this was expected for the model $\mathrm{B}$, leading to the formation of a massive bulge. However, the B model MD does not agree either with the metal-poor component of the observed MD (population A). It peaks around $[\mathrm{Fe} / \mathrm{H}]=-0.40$, close to the mean of the Gaussian representative of the old bulge but is wider than this population and overproduces the fraction of metal-poor stars with respect to the actual observed MD.

The observed and model F MDs peak at distinct metallicities $([\mathrm{Fe} / \mathrm{H}] \sim+0.3$ and $\sim-0.2$ for the observed and model $\mathrm{F}$, respectively), but have quite a similar global shape. Indeed, if we artificially shift the model F MD of +0.5 , the differences between the two MD are less pronounced, even if differences in the lowest and highest metallicities regimes persist. The shifted $\mathrm{F}$ MD would overestimate the number of stars at low metallicities $([\mathrm{Fe} / \mathrm{H}]<-0.8$ dex $)$ and underestimate the number of stars at high metallicities $([\mathrm{Fe} / \mathrm{H}]>0.1)$, in addition to showing a more progressive decrease in the number of stars than observed at high metallicities. Furthermore, we cannot explain why such a high shift $(+0.5)$ in mean metallicity would be required. Uncertainties in the stellar yields adopted can not explain such a large difference. To obtain such metal-rich stars, we would suggest that the gas forming the stellar disc (from which the pseudo-bulge is formed) could have been previously enriched. Several factors could have contributed to the enrichment of this gas, but one possibility is enriched gas left over (or blown out) from the old bulge formation.

\subsection{An attempt at modelling the two populations}

Without any satisfactory bulge formation model that could explain the observed MD, we tried to build a simple modelling of the two populations according to their respective formation mechanism suggested by the results of Babusiaux et al. (2010). The metal-poor population has kinematics that show no influence from the Galactic bar, and in addition, we find it to be enriched in $[\mathrm{Mg} / \mathrm{Fe}]$, which reinforces out interpretation of this population as an old spheroid with a rapid time-scale formation. To produce the chemical evolution of this population, we used a simple closed-box model. This model predicts that the metallicity (Z) distribution function (MDF) follows the relation $f(Z)=p^{-1} \mathrm{e}^{-Z / p}$ where $p$ is the stellar yield given by $p=\langle Z\rangle$. For $p$, we adopted the mean values of the Gaussian associated to the metal old population: -0.06 and $-0.30[\mathrm{Mg} / \mathrm{H}]$ and $[\mathrm{Fe} / \mathrm{H}]$. The resulting $[\mathrm{Fe} / \mathrm{H}]$ and $[\mathrm{Mg} / \mathrm{H}]$ metallicities distribution (scaled to $50 \%$ of the total population to only represent population A) are compared to the red clump ones in Fig. 17 (middle panels).

As explained in Babusiaux et al. (2010), the kinematical characteristics of the richest population suggest that it is under the influence of a bar. Adding to this the fact that $[\mathrm{Mg} / \mathrm{Fe}]$ in this population is nearly solar, we suggest a formation on a long time scale through the evolution of the bar, itself originating in disc instability. Within this scenario, population B stars observed in Baade's window would have been ejected from the inner regions of the Galactic disc. We then expect the chemical composition 
A\&A 534, A80 (2011)
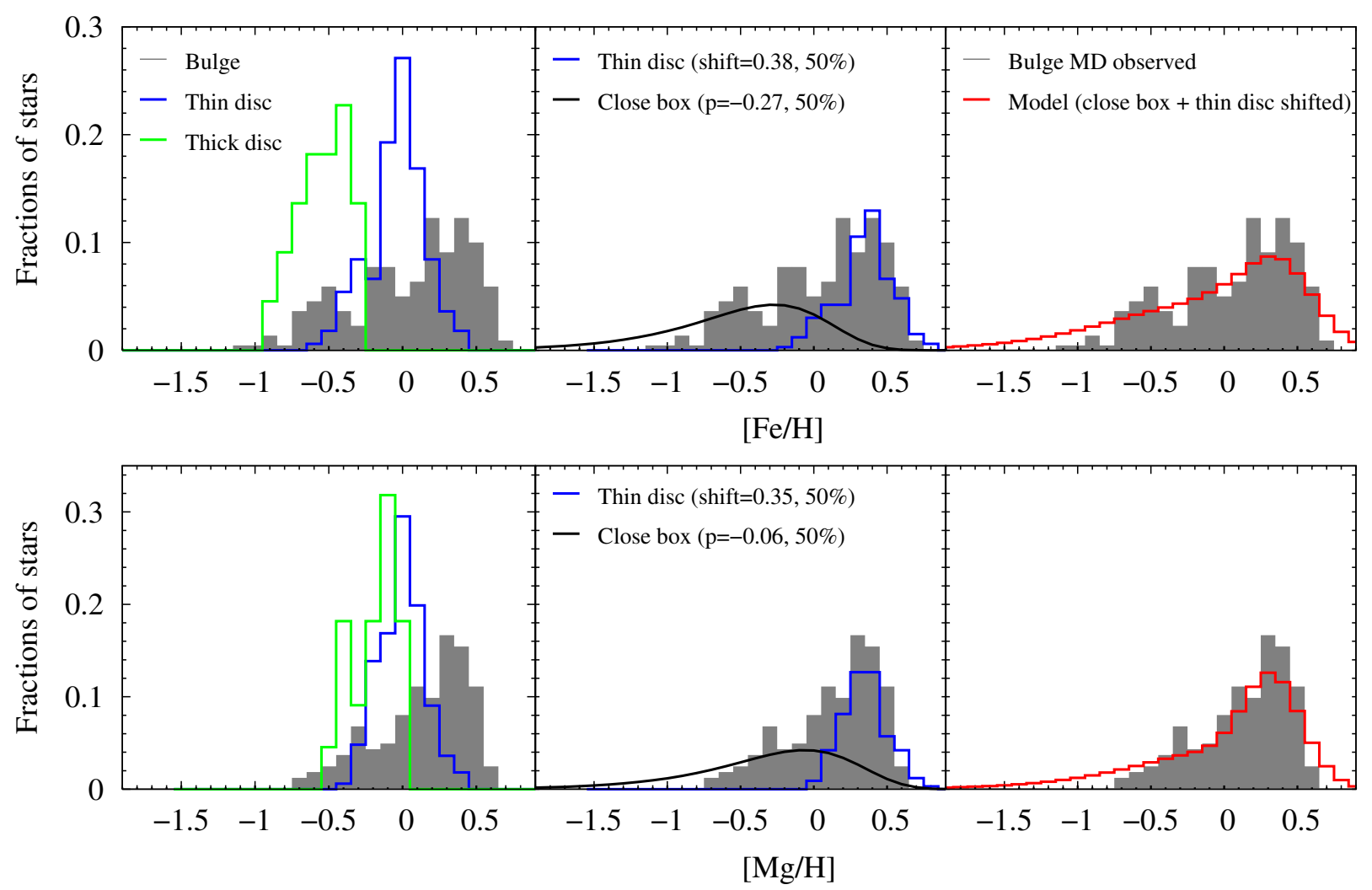

Fig. 17. The left panels show the comparison of the $[\mathrm{Fe} / \mathrm{H}] \mathrm{MD}$ (upper) and $[\mathrm{Mg} / \mathrm{H}] \mathrm{MD}$ (lower) with the thin (blue) and thick (green) discs $\mathrm{MD}$ in the solar neighbourhood from the study of Fuhrmann (2008). The distinction between the three populations is clear from their $[\mathrm{Fe} / \mathrm{H}]$ and $[\mathrm{Mg} / \mathrm{H}]$ $\mathrm{MD}$ in their global shapes, their mean values and the metallicity range they cover. In the middle panels the bulge $[\mathrm{Mg} / \mathrm{H}]$ and $[\mathrm{Fe} / \mathrm{H}] \mathrm{MD}$ are compared to a close box model with a yield $p=-0.27$ and -0.06 for Fe and $\mathrm{Mg}$ respectively (black line) and to the previous thin disc MD shifted by +0.35 (blue) according to the results of the Gaussian separation (see Sect. 4.2 and Table 5). The combination of these two MD convolved are compared to the observed ones (right panels) for Fe (upper) and $\mathrm{Mg}$ (lower). For both elements, the model MD shows a very good agreement with the observed one. A small difference (compared to the relative errors) exists on the high metallicities tails: the high metallicity tail of the Mg and Fe MD decrease more sharply than the model distributions.

of these stars to be the same as the one of the old inner disc at the time when the bar formed. However, as already mentioned, little is known about the inner regions of the Galactic disc. A metallicity gradient has been observed in young populations (OB stars, HII regions, Cepheids), but observational constrains of the old populations are missing. To reproduce the MD of the richest population, we adopted the MD of the solar neighbourhood from Fuhrmann (2008), scaled it to $50 \%$ of the total sample (to represent population B only), and shifted toward higher metallicity in order to reproduce the possible gradient in the Galactic disc. The Fuhrmann (2008) Fe and Mg metallicity distributions are symmetric around mean values of $[\mathrm{Fe} / \mathrm{H}]=-0.03 \pm 0.01$ and $[\mathrm{Mg} / \mathrm{H}]=0.00 \pm 0.01$, and narrower than the bulge MD (see Fig. 17, left panels). In the middle panels of Fig. 17, we plot the Fuhrmann (2008) Fe and Mg MD scaled to 50\% of the total bulge population, and shifted by +0.35 for both $\mathrm{Fe}$ and $\mathrm{Mg}$, so that their mean values coincide with those of the corresponding Gaussian components. These $\mathrm{Mg}$ and $\mathrm{Fe} \mathrm{MD}$ shifted disc components seem to be good candidates for reproducing the observed bulge metal-rich component. The main difference (more pronounced for Fe than for Mg MD) appears at very high metallicities, where the observed bulge MD shows a sharper cutoff than in the shifted disc. Assuming that this mock disc would originate in the very inner parts of the Galactic disc, around $8 \mathrm{kpc}$ from the Sun, the shifts applied to the local thin disc MD of Fuhrmann (2008) correspond to gradients of $-0.047 \mathrm{dex} \mathrm{kpc}{ }^{-1}$ for $\mathrm{Fe}$ and $-0.043 \mathrm{dex} \mathrm{kpc}^{-1}$ for $\mathrm{Mg}$. These gradients are similar to the gradients observed in the youngest populations of the inner and outer discs (Daflon \& Cunha 2004). However, in our case we are not interested in the value of the present day gradient in the Galactic disc but rather what it was in the past when the stars that we now see in the pseudo-bulge would have formed. Recent observations of planetary nebulae and open clusters in the Galatic disc (see Maciel \& Costa 2009, and references therein) suggest a flattening of the metallicity gradients with time that is inconsistent with the gradient we derived. The chemical evolution models of the disc assuming an inside-out formation scenario (Cescutti et al. 2007; Chiappini et al. 2001) predict gradients at the present day whose values agree with the observations of young and intermediate-age populations of the disc. But, these models also predict that the gradients were flatter at in the past. Cescutti et al. (2007, priv. comm.) found that 9 Gyr ago, gradients were only of $-0.014 \mathrm{dex} \mathrm{kpc}^{-1}$ for Fe and of $-0.017 \mathrm{dex} \mathrm{kpc}^{-1}$ (in the range of galactocentric distances from 4 to $14 \mathrm{kpc}$ ). These values, if extended, would also be inconsistent with our findings.

In Fig. 17, we plotted the sum of the two modelled MD associated to the metal-poor and metal-rich components. The MD predicted by the simple model have been convolved with the observed mean uncertainties for our sample (Fig. 6). The internal errors of the Fuhrmann (2008) data being much lower than the observed one, the disc MD have also been convolved with a Gaussian with $\sigma=0.18$ for $\mathrm{Fe}$ and $\sigma=0.12$ for $\mathrm{Mg}$. For both elements, the combined MD show fairly good agreement with the observed ones. The main differences concern the metal-poor tail and the richest part of the distributions. 
In the very metal-poor regime $([\mathrm{Fe} / \mathrm{H}]<-0.7$ and $[\mathrm{Mg} / \mathrm{Fe}]<$ $-0.5)$, the closed box model predicts more stars than what is observed. The same lack is observed in the solar neighbourhood thin disc and is well known as the $\mathrm{G}$ dwarf problem. For our red clump sample, it is not likely that this difference could come from a selection effect, at least down to $[\mathrm{Fe} / \mathrm{H}]=-1.3$ or even -1.5 (see Sect. 2.2). As for the local disc, the $\mathrm{G}$ dwarf problem in the bulge could be partly or totally solved by including an infall of primordial gas in chemical evolution models. Additional observations of the bulge in the very metal-poor regime would also help answer this question.

At higher metallicities, the observed MDs show a more pronounced peak and a sharper decrease than the shifted MD of the thin disc. These differences are insensitive to changes up to 0.05 dex on the adopted shifts for $\mathrm{Mg}$ and Fe but could be reduced by decreasing the adopted width of the convolution (reproducing observed errors) down to an unrealistic level of 0.10 . In Sect. 4.1.3, we argued that the high-metallicity tail of the iron MD may be biased at the highest metallicity stars, in a direction that is not yet very clear. Experiments with synthetic spectra suggest that we may overestimate the metallicities of super-solar metallicity stars by up to 0.15 dex, whereas the estimated errors and sharpness of the decrease in the metal-rich tail may be read as a hint that the highest metallicities are underestimated.

\section{Summary and conclusions}

We analysed a sample of 219 bulge red clump stars in Baade's window from $R=20000$ resolution spectra obtained with FLAMES/GIRAFFE at the VLT. This sample was selected based on the location of stars in the colour-magnitude diagram, in order to minimise the contamination by other Galactic components $(<10 \%$ from the latest population synthesis Besançon model (Robin et al. 2003; Picaud \& Robin 2004), so it is a good tracer of the bulge metallicity distribution (MD) in Baade's window.

For these stars, we used an automatic procedure to derive the stellar parameters and $\mathrm{Fe}$ abundances differentially with respect to the metal-rich local reference star $\mu$ Leo. For a subsample of 162 stars (built by excluding stars with high uncertainties on $[\mathrm{Fe} / \mathrm{H}])$, we also derived $[\mathrm{Mg} / \mathrm{H}]$ from spectral synthesis around the $\mathrm{Mg}$ I triplet at $\lambda 6319 \AA$.

The $\mathrm{Fe}$ and $\mathrm{Mg}$ metallicity distributions are both asymmetric with mean values of $+0.05 \pm 0.03$ and $+0.14 \pm 0.03$ and median values of 0.16 and 0.21 , respectively. They show a low proportion of stars at low metallicities $(<3 \%$ with $[\mathrm{Fe} / \mathrm{H}]<-0.7$ or $[\mathrm{Mg} / \mathrm{H}]<-0.4)$, extending only down to $[\mathrm{Fe} / \mathrm{H}]=-1.1$ or $[\mathrm{Mg} / \mathrm{H}]=-0.7$. The iron MD is reproduced well neither by the predictions of the chemical models of the bulge available in the literature (Ballero et al. 2007a,b) nor by models that add dynamical aspects to chemical prescriptions such as the one of Immeli et al. (2004).

The decomposition of the observed Fe and $\mathrm{Mg}$ MDs into Gaussian components has revealed two populations of equal sizes $(50 \%$ each): a metal-poor component centred on $[\mathrm{Fe} / \mathrm{H}]=$ -0.30 and $[\mathrm{Mg} / \mathrm{H}]=-0.06$ with a large dispersion and a narrow metal-rich component centred around $[\mathrm{Fe} / \mathrm{H}]=0.32$ and $[\mathrm{Mg} / \mathrm{H}]=+0.35$. The metal-poor component shows high $[\mathrm{Mg} / \mathrm{Fe}]$ ratios (around 0.3 ), whereas stars in the metal-rich component are found to have nearly solar ratios. These ratios are in perfect agreement with the recent work of Gonzalez et al. (2011) on $\sim 650$ RGB stars at four different locations in the bulge. Babusiaux et al. (2010) also find kinematical differences between the two components: the metal-poor component shows kinematics compatible with an old spheroid, whereas the metalrich component is consistent with a population supporting a bar. In view of their chemical and kinematical properties, we suggest different formation scenarios for the two populations: a rapid formation time scale as an old spheroid for the metal-poor component (old bulge) and for the metal-rich component, a formation over a longer time scale driven by the evolution of the bar (pseudo-bulge). This component represents $50 \%$ of the total Baade's window population, and has an old age (as indicated by deep colour-magnitude diagrams, see e.g., Clarkson et al. 2008), indicating that a fairly massive disc was present some $10 \mathrm{Gyr}$ ago, which may turn out to be a strong constraint on models of disc formation. It is interesting that the local thick disc and old bulge display the same $[\mathrm{Mg} / \mathrm{Fe}]$ enhancement, pointing at a similarly rapid star formation process. At this point, it is impossible to further constrain the relationship that the old bulge has with the thick disc, both because constraints are lacking in the central parts of the thick disc (both chemistry and kinematics) and because our bulge sample is located on the minor axis so we have very poor constraints on the rotation or the disc-like nature of this component. We do however, note that in their recent study, Bensby et al. (2010a) finds that the inner parts of the thick disc are chemically very similar to those in the solar neighbourhood, lending strong support to the idea that the metal-poor bulge is indeed chemically related to the thick disc. We also note that the numerous metal-rich globular clusters of the Milky Way, which are concentrated towards the inner parts of the galaxy, would be classified as old bulge in our scheme (see e.g., Bica et al. 2006, who shows that clusters with $[\mathrm{Fe} / \mathrm{H}]<-0.75$ are confined within $2 \mathrm{kpc}$ of the centre). It is interesting that even the most metal-rich globular cluster known so far (NGC 6528, with $[\mathrm{Fe} / \mathrm{H}]=-0.2$, Zoccali et al. 2004) belongs the metal-poor bulge component as defined here.

We finally attempted modelling the observations by combining a simple closed box model to represent the metal poor population, and the MD of the local thin disc (as observed by Fuhrmann 2008) shifted in metallicity to represent the metalrich population. The resulting combined MD shows good agreement with the red clump MD on the whole metallicity range except for the very metal-poor and very metal-rich regimes, where more stars are predicted than observed. The bulge (old bulge) thus seems to exhibit the G dwarf problem. The shift we had to apply to the local thin disc MD to fit the metal-rich population is, however, quite large (although plausible) when compared with plausible values of the gradients in the early Galactic disc. One possibility could be that these stars were born in a pre-enriched environment, enriched by the remnant of the old bulge.

The scenario(s) proposed here should now be turned into proper modelling (chemical and kinematical) of the bulge that would include two formations events (linked or not), to evaluate their viability. Expanding similar combined chemical and kinematical studies of large samples towards various directions in the Galactic bulge would also be of great help for answering these questions. In particular, probing the bulge at lower latitudes and at various longitudes should trace the relative importance of the metal-rich and metal-poor populations and definitively establish their proposed different (kinematical and chemical) nature as an old bulge and pseudo-bulge. Because these regions are much more heavily obscured than Baade's window, this kind of observation will have to be carried out in the infrared. Finally, our group has undertaken similar red clump observations of the inner parts of the Galactic disc which are expected to shed new light on the true relation between the disc and the pseudo-bulge. 
Acknowledgements. We thank Bertrand Plez for kindly providing molecular line lists and the synthetic spectrum code. M.Z. and D.M. are supported by FONDAP Centre for Astrophysics 15010003, the BASAL Centre for Astrophysics and Associated Technologies PFB-06, the FONDECYT 1110393 and 1090213, and the Milky Way Millennium Nucleus from the Ministry of Economy ICM grant P07-021-F.

\section{References}

Aguerri, J. A. L., Balcells, M., \& Peletier, R. F. 2001, A\&A, 367, 428 Alonso, A., Arribas, S., \& Martínez-Roger, C. 1999, A\&AS, 140, 261 Alvarez, R., \& Plez, B. 1998, A\&A, 330, 1109

Alves-Brito, A., Meléndez, J., Asplund, M., Ramírez, I., \& Yong, D. 2010, A\&A, 513, A35

Arenou, F. 1993, Ph.D. Thesis, Observatoire de Paris, CNRS

Babusiaux, C., \& Gilmore, G. 2005, MNRAS, 358, 1309

Babusiaux, C., Gomez, A., Hill, V., et al. 2010, A\&A, 519, A77

Ballero, S. K., Kroupa, P., \& Matteucci, F. 2007a, A\&A, 467, 117

Ballero, S. K., Matteucci, F., Origlia, L., \& Rich, R. M. 2007b, A\&A, 467, 123

Bensby, T., Feltzing, S., \& Lundström, I. 2004, A\&A, 415, 155

Bensby, T., Feltzing, S., Lundström, I., \& Ilyin, I. 2005, A\&A, 433, 185

Bensby, T., Zenn, A. R., Oey, M. S., \& Feltzing, S. 2007, ApJ, 663, L13

Bensby, T., Feltzing, S., Johnson, J. A., et al. 2009a, ApJ, 699, L174

Bensby, T., Johnson, J. A., Cohen, J., et al. 2009b, A\&A, 499, 737

Bensby, T., Alves-Brito, A., Oey, M. S., Yong, D., \& Meléndez, J. 2010a, A\&A, 516, L13

Bensby, T., Feltzing, S., Johnson, J. A., et al. 2010b, A\&A, 512, A41

Blecha, A., Cayatte, V., North, P., Royer, F., \& Simond, G. 2000, in Optical and IR Telescope Instrumentation and Detectors, ed. M. Iye, \& A. F. Moorwood, Proc. SPIE, 4008, 467

Bowman, A. W., \& Azzalini, A. 1997, Applied smoothing techniques for data analysis: the kernel approach with S-plus illustrations, Oxford statistical science series No. 18 (Clarendon Press)

Celeux, G., \& Diebolt, J. 1986, Rev. Stat. Appl., 34, 35

Cescutti, G., Matteucci, F., François, P., \& Chiappini, C. 2007, A\&A, 462, 943

Chiappini, C., Matteucci, F., \& Romano, D. 2001, ApJ, 554, 1044

Clarkson, W., Sahu, K., Anderson, J., et al. 2008, ApJ, 684, 1110

Cohen, J. G., Huang, W., Udalski, A., Gould, A., \& Johnson, J. A. 2008, ApJ, 682,1029

Cohen, J. G., Thompson, I. B., Sumi, T., et al. 2009, ApJ, 699, 66

Combes, F., \& Sanders, R. H. 1981, A\&A, 96, 164

Daflon, S., \& Cunha, K. 2004, ApJ, 617, 1115

de Vaucouleurs, G. 1964, in The Galaxy and the Magellanic Clouds, ed.

F. J. Kerr, IAU Symp., 20, 195

Donati, J.-F., Semel, M., Carter, B. D., Rees, D. E., \& Collier Cameron, A. 1997, MNRAS, 291, 658

Dwek, E., Arendt, R. G., Hauser, M. G., et al. 1995, ApJ, 445, 716

Eggen, O. J., Lynden-Bell, D., \& Sandage, A. R. 1962, ApJ, 136, 748

Epchtein, N., de Batz, B., Capoani, L., et al. 1997, The Messenger, 87, 27

Epstein, C. R., Johnson, J. A., Dong, S., et al. 2010, ApJ, 709, 447

Feltzing, S., \& Gilmore, G. 2000, A\&A, 355, 949

Fuhrmann, K. 2008, MNRAS, 384, 173

Fulbright, J. P., McWilliam, A., \& Rich, R. M. 2006, ApJ, 636, 821

Fulbright, J. P., McWilliam, A., \& Rich, R. M. 2007, ApJ, 661, 1152

Gerhard, O. 2006, in EAS Pub. Ser. 20, ed. G. A. Mamon, F. Combes, C. Deffayet, \& B. Fort, 89

Girardi, L., Bressan, A., Bertelli, G., \& Chiosi, C. 2000, A\&AS, 141, 371

Glass, I. S. 1999, Handbook of Infrared Astronomy (Cambridge University Press)

Gonzalez, O. A., Rejkuba, M., Zoccali, M., et al. 2011, A\&A, 530, A54

Gustafsson, B., Edvardsson, B., Eriksson, K., et al. 2008, A\&A, 486, 951
Howard, C. D., Rich, R. M., Reitzel, D. B., et al. 2008, ApJ, 688, 1060 Howard, C. D., Rich, R. M., Clarkson, W., et al. 2009, ApJ, 702, L153 Ibata, R. A., \& Gilmore, G. F. 1995a, MNRAS, 275, 591

Ibata, R. A., \& Gilmore, G. F. 1995b, MNRAS, 275, 605

Immeli, A., Samland, M., Gerhard, O., \& Westera, P. 2004, A\&A, 413, 547

Johnson, J. A., Gal-Yam, A., Leonard, D. C., et al. 2007, ApJ, 655, L33

Johnson, J. A., Gaudi, B. S., Sumi, T., Bond, I. A., \& Gould, A. 2008, ApJ, 685, 508

Kormendy, J., \& Kennicutt, Jr., R. C. 2004, ARA\&A, 42, 603

Kroupa, P. 2001, MNRAS, 322, 231

Kuijken, K., \& Rich, R. M. 2002, AJ, 124, 2054

Kupka, F., Piskunov, N., Ryabchikova, T. A., Stempels, H. C., \& Weiss, W. W. 1999, A\&AS, 138, 119

Lecureur, A., Hill, V., Zoccali, M., et al. 2007, A\&A, 465, 799

Lucy, L. B. 1974, AJ, 79, 745

Lucy, L. B. 1994, A\&A, 289, 983

Maciel, W. J., \& Costa, R. D. D. 2009, in IAU Symp. 254, ed. J. Andersen, J. Bland-Hawthorn, \& B. Nordström, 38P

Magain, P. 1984, A\&A, 134, 189

McWilliam, A., \& Rich, R. M. 1994, ApJS, 91, 749

McWilliam, A., Preston, G. W., Sneden, C., \& Searle, L. 1995, AJ, 109, 2757

Meléndez, J., Asplund, M., Alves-Brito, A., et al. 2008, A\&A, 484, L21

Minniti, D. 1996, ApJ, 459, 175

Mishenina, T. V., Bienaymé, O., Gorbaneva, T. I., et al. 2006, A\&A, 456, 1109

Nakasato, N., \& Nomoto, K. 2003, ApJ, 588, 842

Nishiyama, S., Nagata, T., Sato, S., et al. 2006, ApJ, 647, 1093

Noguchi, M. 1999, ApJ, 514, 77

Ortolani, S., Renzini, A., Gilmozzi, R., et al. 1995, Nature, 377, 701

Paczynski, B., Udalski, A., Szymanski, M., et al. 1999, Acta Astron., 49, 319

Pfenniger, D., \& Norman, C. 1990, ApJ, 363, 391

Picaud, S., \& Robin, A. C. 2004, A\&A, 428, 891

Plez, B. 1998, A\&A, 337, 495

Raha, N., Sellwood, J. A., James, R. A., \& Kahn, F. D. 1991, Nature, 352, 411

Ralchenko, Y., Kramida, A. E., Reader, J., \& NIST ASD Team 2008, http:// physics.nist.gov/asd3, National Institute of Standards and Technology, Gaithersburg, MD

Ramírez, I., \& Meléndez, J. 2005, ApJ, 626, 465

Rattenbury, N. J., Mao, S., Sumi, T., \& Smith, M. C. 2007, MNRAS, 378, 1064

Reddy, B. E., Lambert, D. L., \& Allende Prieto, C. 2006, MNRAS, 367, 1329

Reid, M. J. 1993, ARA\&A, 31, 345

Rich, R. M. 1988, AJ, 95, 828

Rich, R. M., \& Origlia, L. 2005, ApJ, 634, 1293

Rich, R. M., Origlia, L., \& Valenti, E. 2007, ApJ, 665, L119

Richardson, W. H. 1972, J. Opt. Soc. Am., 62, 55

Robin, A. C., Reylé, C., Derrière, S., \& Picaud, S. 2003, A\&A, 409, 523

Royer, F., Zorec, J., \& Gómez, A. E. 2007, A\&A, 463, 671

Sadler, E. M., Rich, R. M., \& Terndrup, D. M. 1996, AJ, 112, 171

Scannapieco, C., \& Tissera, P. B. 2003, MNRAS, 338, 880

Sheather, S. J., \& Jones, M. C. 1991, J. R. Statist. Soc. B, 53, 683

Skrutskie, M. F., Cutri, R. M., Stiening, R., et al. 2006, AJ, 131, 1163

Soto, M., Rich, R. M., \& Kuijken, K. 2007, ApJ, 665, L31

Spite, M. 1967, Annales d'Astrophysique, 30, 211

Stetson, P. B., \& Pancino, E. 2008, PASP, 120, 1332

Terndrup, D. M., Popowski, P., Gould, A., Rich, R. M., \& Sadler, E. M. 1998, AJ, 115, 1476

Udalski, A., Kubiak, M., \& Szymanski, M. 1997, Acta Astron., 47, 319

Weidner, C., \& Kroupa, P. 2005, ApJ, 625, 754

Zhao, H., Spergel, D. N., \& Rich, R. M. 1994, AJ, 108, 2154

Zoccali, M., Renzini, A., Ortolani, S., et al. 2003, A\&A, 399, 931

Zoccali, M., Barbuy, B., Hill, V., et al. 2004, A\&A, 423, 507

Zoccali, M., Lecureur, A., Barbuy, B., et al. 2006, A\&A, 457, L1

Zoccali, M., Hill, V., Lecureur, A., et al. 2008, A\&A, 486, 177 